\title{
Dynamically generated reduction of the mean photon number in the Dicke model
}

\author{
Michael Tomka, ${ }^{1, *}$ Dionys Baeriswyl, ${ }^{1}$ and Vladimir Gritsev ${ }^{1,2}$ \\ ${ }^{1}$ Physics Department, University of Fribourg, Chemin du Musée 3, 1700 Fribourg, Switzerland \\ ${ }^{2}$ Institute for Theoretical Physics, University of Amsterdam, Science Park 904, Postbus 94485, 1098 XH Amsterdam, The Netherlands
}

(Received 26 October 2012; published 1 November 2013)

\begin{abstract}
We study the dynamics of a driven Dicke model, where the collective spin is rotated with a constant velocity around a fixed axis. The time evolution of the mean photon number and of the atomic inversion is calculated using, on the one hand, a numerical technique for the quantum dynamics of a small number of two-level atoms and, on the other hand, time-dependent mean-field theory for the limit of a large number of atoms. We observe a reduction in the mean photon number compared to its equilibrium value. This dynamically generated darkness is particularly pronounced slightly above the transition to a superradiant phase. We attribute the effect to a slowing-down of the motion in the classical limit of a large ensemble and to an interplay of dynamic and geometric phases in the quantum case.
\end{abstract}

DOI: 10.1103/PhysRevA.88.053801

PACS number(s): 42.50.Pq, 05.30.Rt, 03.65.Vf, 42.50.Nn

\section{INTRODUCTION}

The Dicke model [1,2] describes $N$ two-level atoms interacting with a single-mode radiation field through the dipole coupling. If the atoms are confined to a region of space which is much smaller than the wavelength of the radiation field, the two-level emitters behave as a single large spin. The model is a paradigm for the collective behavior of matter interacting with light. In the thermodynamic limit, $N \rightarrow \infty$, there is a transition to a superradiant phase at a critical coupling strength [3-5]. The order parameter can be chosen to be the photon density, which vanishes in the normal phase and is finite in the superradiant phase. Alternatively, one may also use the fraction of excited atoms, which behaves similarly to the photon density. Recently the emphasis was on the quantum phase transition at zero temperature. It was shown that the entanglement between photons and atoms diverges upon approaching the quantum critical point [6]. Interestingly, for finite $N$ clear signatures of the onset of quantum chaos are also found for couplings at and not too far above the quantum critical point [7].

The existence of this phase transition in a real material was called seriously into question some time ago. It was argued that adding the term proportional to $A^{2}$ to the Dicke model, where $A$ is the vector potential of the electromagnetic field, would eliminate the equilibrium transition [8]. However, in a more recent study it was pointed out that within a consistent treatment of interactions between excited atoms a transition would still occur, although perhaps of a different type than originally thought $[9,10]$. A different route would be to realize the Dicke model in a nonequilibrium setting of cavity quantum electrodynamics [11]. An experimental breakthrough was achieved with a high-quality cavity hosting a Bose-Einstein condensate [12]. This setup not only is considered to be a realization of the Dicke model, but also produced data showing evidence for a superradiant phase transition and other collective behaviors [13].

The combination of cold atomic gases and cavity quantum electrodynamics has the advantage that parameter values

*michael.tomka@unifr.ch can be tuned with a high precision and even controlled in real time. This type of experiment has stimulated studies of nonequilibrium effects and, especially, their interplay with collective phenomena, for instance, in the vicinity of a quantum phase transition. A fundamental problem is the evolution of an initial state-or density matrix-in a model without dissipation. For the (time-independent) Dicke model an approach to equilibrium has been found, governed by a competition between classical chaos and quantum diffusion [14]. The Dicke model turns into a many-body Landau-Zener model if both atomic and photonic excitation energies become time dependent. In contrast to the usual Landau-Zener model with a single two-level system, where adiabatic evolution — and thus no tunneling - is easily achieved for slow enough driving, this becomes very difficult in a problem involving many two-level systems [15]. At the critical point adiabatic evolution appears to be impossible [16]. A different scenario is obtained if the coupling constant is time dependent. Here a rich new phase diagram including metastable phases and first-order transitions has been reported [17].

In several papers dealing with the dynamics of the Dicke model the rotating wave approximation has been used, where the emission of a photon is accompanied by the transition of an excited atom to its ground state and the absorption of a photon is linked to the excitation of an atom. This yields an integrable model where the dynamics is not chaotic. In one study the evolution after an initial quench has been found either to lead to a constant photon density at long times or to produce persistent oscillations with several incommensurable frequencies [18]. Other studies investigated the conditions under which a classical treatment of the dynamics is valid $[19,20]$.

In this paper we consider a specific time-dependent coupling where the spin operator is rotated with a time-dependent angle. This "rotated Dicke Hamiltonian" can be generated by a unitary transformation starting from the time-independent Hamiltonian. Therefore the energy eigenvalues do not depend on time, in contrast to the study mentioned above [17]. Nevertheless, the evolution is highly nontrivial and leads to qualitatively new effects at and slightly above the equilibrium quantum critical point. We are particularly interested in the thermodynamic limit, where a time-dependent mean-field 
theory is expected to yield exact results for the dynamics. We develop this theory on the basis of a coherent state representation of both photon and spin degrees of freedom. We obtain a classical dynamical system of two oscillators, which are coupled by a nonlinear time-dependent term. Alternatively, this classical limit can also be reached in the framework of the Holstein-Primakoff transformation. Once the classical dynamical system is established, it is straightforward to calculate the relevant physical quantities, such as the (time-dependent) photon density. For finite $N$ we complement the mean-field calculations with a numerical procedure where the quantum evolution for a specific initial state is computed for the rotated Dicke Hamiltonian. For large $N$, these numerical results approach the mean-field predictions, as expected.

In our calculations we kept all coupling terms of the Dicke model and did not use the rotating wave approximation. At weak coupling one does not find noticeable effects of the terms neglected by the rotating wave approximation, but at and above the quantum critical point the differences between integrable and nonintegrable cases show up clearly, for instance, through the onset of chaos in the latter case.

The following general picture emerges from our calculations. The rotation by a time-dependent angle leads to an upshift of the critical coupling strength above which a finite photon density is produced spontaneously. If the system is prepared in the ground state of the (time-independent) Dicke model slightly above its quantum critical point, i.e., with a finite photon density, an interesting darkening effect occurs after the rotation is switched on. A clear minimum in the photon density is found as a function of the coupling strength for a fixed driving velocity or, even more pronounced, as a function of driving velocity for a fixed coupling strength. The location of the minimum defines a dynamical critical line in the coupling-versus-velocity plane. The effect can be readily understood as a slowing-down of the nonlinear dynamics in the classical limit. For a small number of two-level systems, where the evolution has to be treated quantum mechanically, we find that the geometric phase associated with the time-dependent state of the system plays a crucial role in this dynamically generated darkness.

The paper is organized as follows. Section II gives a short review of the standard (time-independent) Dicke model. The time-dependent Dicke model is introduced in Sec. III. The rotation by a time-dependent angle can be partly compensated by a transformation to a corotating frame. The numerical procedure used for treating the quantum dynamics of a limited number of two-level systems is also explained. Section IV presents the time-dependent mean-field theory using coherent states for both the spin and the photons. The stationary states in the corotating frame yield a modified phase diagram, compared to that of the time-independent Dicke model. Two linear modes describe motions close to equilibrium, one of which softens upon approaching criticality. However, in the vicinity of the critical point the dynamics is highly nonlinear. This becomes clear in Sec. V, where the evolution of the system is studied after the rotation is suddenly switched on. The phenomenon of dynamically generated darkness, observed close to criticality, is interpreted as a slowing-down of the motion, in analogy to an effect experienced by a particle moving in the Mexican hat potential. Section VI shows that in the quantum limit (small number of two-level systems) the minimum in the photon density disappears if the geometric phase is removed by hand, which suggests that the darkening effect results from an interplay of geometric and dynamic phases. A brief summary is presented in the concluding Sec. VII. Appendixes A and B give some details on the linearized classical dynamics and the slowing-down in the Mexican hat, respectively.

\section{TIME-INDEPENDENT DICKE MODEL}

The Dicke model takes into account a single radiation mode and reduces the atoms to two-level systems, described by the Pauli matrices $\hat{\sigma}_{i}^{\mu}, \mu=z, \pm, i=1, \ldots, N$. The parameters of the model are the photon frequency $\omega$, the level splitting $\omega_{0}$ (we choose $\hbar=1$ ), and the coupling constant $\lambda$. The Hamiltonian reads

$$
\hat{H}_{\mathrm{D}}=\omega_{0} \hat{J}_{z}+\omega \hat{a}^{\dagger} \hat{a}+\frac{\lambda}{\sqrt{N}}\left(\hat{a}^{\dagger}+\hat{a}\right)\left(\hat{J}_{+}+\hat{J}_{-}\right),
$$

where $\hat{J}_{\mu}=\sum_{i=1}^{N} \hat{\sigma}_{i}^{\mu} / 2$ are collective atomic operators satisfying the commutation relations of the angular momentum,

$$
\left[\hat{J}_{+}, \hat{J}_{-}\right]=2 \hat{J}_{z}, \quad\left[\hat{J}_{z}, \hat{J}_{ \pm}\right]= \pm \hat{J}_{ \pm},
$$

and $\hat{a}^{\dagger}$ and $\hat{a}$ are photon creation and annihilation operators with bosonic commutation relations.

The Hilbert space of the two-level systems is spanned by the Dicke states $|j, m\rangle$, which are eigenstates of $\hat{\mathbf{J}}^{2}$ and $\hat{J}_{z}$ with eigenvalues $j(j+1)$ and $m$, respectively, $j$ being a nonnegative integer or a positive half-integer and $m$ being restricted to $-j \leqslant m \leqslant j . \hat{H}_{\mathrm{D}}$ commutes with $\hat{\mathbf{J}}^{2}$ and is therefore block-diagonal with respect to the Dicke states. As in Ref. [7] we fix $j$ to its maximal value $j=N / 2$. This is a natural choice because the ground state for $\lambda=0$ belongs to this subspace. The collection of two-level atoms can then be interpreted as a large "spin" of magnitude $j=N / 2$. For the photons we use the basis of Fock states $|n\rangle$, with $n=0,1,2,3, \ldots$, defined by

$$
\hat{a}^{\dagger}|n\rangle=\sqrt{n+1}|n+1\rangle, \quad \hat{a}|n\rangle=\sqrt{n}|n-1\rangle .
$$

The Dicke Hamiltonian $\hat{H}_{\mathrm{D}}$ has an important symmetry: it is invariant under a parity operation. To see this, we introduce the unitary transformation

$$
\hat{U}(\varphi)=\exp \left[i \varphi \hat{N}_{\mathrm{ex}}\right]
$$

where the operator $\hat{N}_{\text {ex }}=\hat{a}^{\dagger} \hat{a}+\hat{J}_{z}+j$ counts the number of excited quanta in the system. In view of the relations

$$
\hat{U} \hat{a} \hat{U}^{\dagger}=e^{i \varphi} \hat{a}, \quad \hat{U} \hat{J}_{+} \hat{U}^{\dagger}=e^{i \varphi} \hat{J}_{+}
$$

the transformed Dicke Hamiltonian is given by

$$
\begin{aligned}
\hat{U} \hat{H}_{\mathrm{D}} \hat{U}^{\dagger}= & \omega_{0} \hat{J}_{z}+\omega \hat{a}^{\dagger} \hat{a} \\
& +\frac{\lambda}{\sqrt{2 j}}\left(\hat{a}^{\dagger} \hat{J}_{+} e^{2 i \varphi}+\hat{a}^{\dagger} \hat{J}_{-}+\hat{a} \hat{J}_{+}+\hat{a} \hat{J}_{-} e^{-2 i \varphi}\right)
\end{aligned}
$$

and thus remains invariant if $\varphi$ is a multiple of $\pi$. Therefore the Dicke model is symmetric under the parity transformation defined as

$$
\hat{\Pi}=\hat{U}(\pi)=\exp \left[i \pi \hat{N}_{\mathrm{ex}}\right]
$$


The eigenvalues of $\hat{\Pi}$ are $e^{i \pi(n+m+j)}= \pm 1$. Due to this $\mathbb{Z}_{2}$ symmetry the Hilbert space breaks up into two disjoint pieces which are not coupled by the Hamiltonian: one with an even, and the other with an odd, excitation number $n+m+j$.

The Dicke model, (1), has a quantum phase transition in the thermodynamic limit $N \rightarrow \infty(j \rightarrow \infty)$ at the quantum critical point $\lambda_{c}^{0}=\sqrt{\omega \omega_{0}} / 2$. Both the mean photon number $\left\langle\hat{a}^{\dagger} \hat{a}\right\rangle / j$ and the atomic inversion $\left(\left\langle\hat{J}_{z}\right\rangle+j\right) / j$ vanish for $\lambda<$ $\lambda_{c}^{0}$ and are finite for $\lambda>\lambda_{c}^{0}$. Numerical results for finite $j$ show that the thermodynamic limit is approached very quickly. Already the curves for $j=5$ follow the $j \rightarrow \infty$ results rather closely, except in a small region around the critical point [7].

Equation (6) shows that only the counter-rotating terms are affected by the transformation $\hat{U}(\varphi)$. Within the rotating wave approximation, where these terms are neglected, the Dicke Hamiltonian does not depend on the angle $\varphi$ and therefore has $\mathbb{U}(1)$ symmetry. Nevertheless, there is again a quantum phase transition with a similar behavior for the mean photon number (and also for the atomic inversion), except that the critical coupling of the full Dicke model is multiplied by a factor of 2 .

\section{TIME-DEPENDENT DICKE MODEL}

\section{A. Model and model parameters}

The focus of our investigation is on a rotationally driven version of the Dicke model [21]. The unitary transformation

$$
\hat{R}_{z}(t)=\exp \left[-i \phi(t) \hat{J}_{z}\right]
$$

generates a rotation of the spin $\hat{\mathbf{J}}$ (of length $j=N / 2$ ) around the $z$ axis with a time-dependent angle $\phi(t)$. Applying this transformation to the Dicke Hamiltonian, (1), we get

$$
\begin{aligned}
\hat{H}_{\mathrm{RD}}(t)= & \hat{R}_{z}^{\dagger}(t) \hat{H}_{\mathrm{D}} \hat{R}_{z}(t) \\
= & \omega_{0} \hat{J}_{z}+\omega \hat{a}^{\dagger} \hat{a}+\frac{\lambda}{\sqrt{2 j}}\left(\hat{a}^{\dagger}+\hat{a}\right) \\
& \times\left(e^{i \phi(t)} \hat{J}_{+}+e^{-i \phi(t)} \hat{J}_{-}\right) .
\end{aligned}
$$

We choose a linear time dependence,

$$
\phi(t)=\delta_{\phi} t,
$$

so that the two-dimensional vector $(\lambda \cos \phi, \lambda \sin \phi)$ describes a circular motion with constant velocity $\delta_{\phi}$. This is illustrated in Fig. 1, where two distinct paths are shown. The path $\mathcal{C}_{2}$ lies inside the critical surface, defined by the equation $\omega_{0}=4 \lambda^{2} / \omega$ and, thus, probes the normal phase, while the path $\mathcal{C}_{1}$ lies outside and probes the superradiant phase, where intriguing dynamical effects appear.

In the following the analysis is developed for general values of the parameters, but concrete calculations are carried out at resonance, and we choose $\omega=\omega_{0}=1$. In this case there remain two relevant parameters, the coupling strength $\lambda$ and the driving velocity $\delta_{\phi}$.

\section{B. Dynamics}

The rotationally driven Dicke Hamiltonian $\hat{H}_{\mathrm{RD}}(t)$ is obtained by applying a unitary transformation, therefore its eigenvalues are the same as those of the original Hamiltonian $\hat{H}_{\mathrm{D}}$. Nevertheless, the evolution, governed by the time-dependent Schrödinger equation, $i \partial_{t}\left|\psi_{\mathrm{RD}}(t)\right\rangle=$

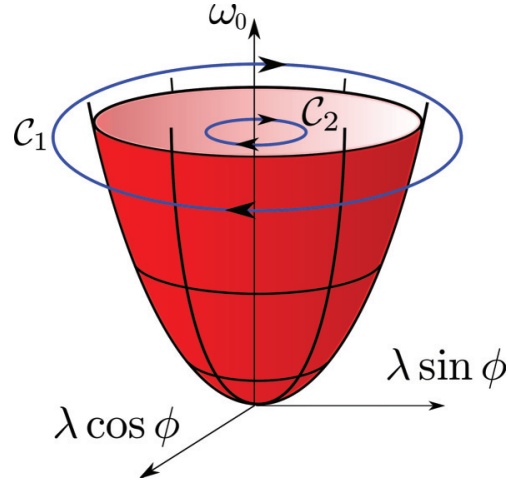

FIG. 1. (Color online) Critical surface of the Dicke model (for the case of a time-independent angle $\phi$ ) and circular paths chosen in the study of the time-dependent Dicke model.

$\hat{H}_{\mathrm{RD}}(t)\left|\psi_{\mathrm{RD}}(t)\right\rangle(\hbar=1)$, will lead to novel results, thanks to the combined effects of geometric and dynamic phases. Our approach is to start with a specific initial state at $t=0$ and to let the system evolve according to the Schrödinger equation up to a time $t>0$, where some quantities are measured. A particularly important quantity is the mean photon number,

$$
n_{\mathrm{ph}}(t)=\frac{1}{j}\left\langle\psi_{\mathrm{RD}}(t)\left|\hat{a}^{\dagger} \hat{a}\right| \psi_{\mathrm{RD}}(t)\right\rangle,
$$

which, in the case of the time-independent Dicke Hamiltonian, can be taken as the order parameter. Alternatively, one may also consider the atomic inversion,

$$
n_{\mathrm{at}}(t)=1+\frac{1}{j}\left\langle\psi_{\mathrm{RD}}(t)\left|\hat{J}_{z}\right| \psi_{\mathrm{RD}}(t)\right\rangle .
$$

The sum of these two quantities is just the average number of excitations,

$$
n_{\mathrm{ex}}(t)=\frac{1}{j}\left\langle\psi_{\mathrm{RD}}(t)\left|\hat{N}_{\mathrm{ex}}\right| \psi_{\mathrm{RD}}(t)\right\rangle .
$$

The analysis is greatly facilitated by a transformation to a corotated state,

$$
\left|\psi_{\mathrm{ROT}}(t)\right\rangle=\hat{R}_{z}(t)\left|\psi_{\mathrm{RD}}(t)\right\rangle,
$$

which evolves according to the Schrödinger equation

$$
i \partial_{t}\left|\psi_{\mathrm{ROT}}(t)\right\rangle=\left(\hat{H}_{\mathrm{D}}+\delta_{\phi} \hat{J}_{z}\right)\left|\psi_{\mathrm{ROT}}(t)\right\rangle .
$$

The operator

$$
\hat{H}_{\mathrm{ROT}}=\hat{H}_{\mathrm{D}}+\delta_{\phi} \hat{J}_{z}
$$

is time independent and has the form of the Dicke Hamiltonian, with $\omega_{0}$ replaced by the renormalized level splitting

$$
\Omega=\omega_{0}+\delta_{\phi} .
$$

Therefore its ground state exhibits a quantum phase transition at $\lambda_{c}=\frac{1}{2} \sqrt{\Omega \omega}$, from a normal phase with a vanishing photon density to a superradiant phase with a finite photon density. The critical value $\lambda_{c}$ is expected to play a central role in the time dependence of physical quantities. 
The expectation value of an operator $\hat{O}$ which commutes with $\hat{J}_{z}$ is not affected by the transformation to a corotated frame,

$$
\begin{aligned}
{\left[\hat{O}, \hat{J}_{z}\right] } & =0 \Rightarrow\left\langle\psi_{\mathrm{RD}}(t)|\hat{O}| \psi_{\mathrm{RD}}(t)\right\rangle \\
& =\left\langle\psi_{\mathrm{ROT}}(t)|\hat{O}| \psi_{\mathrm{ROT}}(t)\right\rangle .
\end{aligned}
$$

This is the case for the quantities mentioned above. If $\left|\psi_{\mathrm{RD}}(0)\right\rangle$ happens to be an eigenstate of $\hat{H}_{\mathrm{ROT}}$, then Eq. (18) implies that $\left\langle\psi_{\mathrm{RD}}(t)|\hat{O}| \psi_{\mathrm{RD}}(t)\right\rangle$ is time independent. Specifically, if $\left|\psi_{\mathrm{RD}}(0)\right\rangle$ is the ground state of $\hat{H}_{\mathrm{ROT}}$, both the photon density, (11), and the fraction of excited atoms, (12), will be time independent and equal to the respective ground state expectation values for $\hat{H}_{\text {ROT }}$.

With coherent states, discussed in the next section, expectation values of the operators $\hat{a}, \hat{a}^{\dagger}$, and $\hat{J}_{ \pm}$are also relevant. While for the photon creation and annihilation operators equality (18) holds, this is no longer true in the case of the operators $\hat{J}_{ \pm}$, for which we get

$$
\left\langle\psi_{\mathrm{RD}}(t)\left|\hat{J}_{ \pm}\right| \psi_{\mathrm{RD}}(t)\right\rangle=e^{ \pm i \phi(t)}\left\langle\psi_{\mathrm{ROT}}(t)\left|\hat{J}_{ \pm}\right| \psi_{\mathrm{ROT}}(t)\right\rangle .
$$

If $\left|\psi_{\mathrm{RD}}(0)\right\rangle$ is an eigenstate of $\hat{H}_{\mathrm{ROT}}$, the vector $\left\langle\psi_{\mathrm{RD}}(t)|\hat{\mathbf{J}}| \psi_{\mathrm{RD}}(t)\right\rangle$ precesses with an angular velocity $\delta_{\phi}$ around the $z$ axis.

\section{Numerical procedure}

We are mostly interested in the thermodynamic limit, $N \rightarrow \infty$, where mean-field theory is expected to produce exact results. In the next section we explain in detail how to construct a time-dependent mean-field theory using coherent states. Calculations for a finite number of two-level atoms $N=2 j$ (or for a pseudospin of finite length $j=N / 2$ ) are nevertheless useful because they allow us both to check the validity of mean-field theory and to study the approach to the thermodynamic limit. In our numerical procedure we truncate the bosonic Hilbert space up to $n_{\mathrm{M}}$ bosons but keep the full $(2 j+1)$-dimensional Hilbert space of the spin. We choose $n_{\mathrm{M}}$ always high enough to assure that the error of the numerical data is on the level of the machine precision. In the corotating frame the evolution is determined by the time-independent Hamiltonian $\hat{H}_{\text {ROT }}$, Eq. (16). To calculate dynamic quantities (18) we use the Chebyshev scheme [22], where the evolution operator for a small time interval $\Delta t$ is expanded in a Chebyshev series,

$$
e^{-i \hat{H}_{\mathrm{ROT}} \Delta t} \approx \sum_{k=0}^{M} a_{k} T_{k}(\hat{h}),
$$

where $T_{k}(\hat{h})$ are the Chebyshev polynomials of order $k$ and $\hat{h}$ is a rescaled Hamiltonian,

$$
\hat{h}=\frac{2 \hat{H}_{\mathrm{ROT}}-\left(E_{\mathrm{Max}}+E_{\mathrm{Min}}\right) \hat{1}}{E_{\mathrm{Max}}-E_{\mathrm{Min}}} .
$$

Here $E_{\mathrm{Max}}$ and $E_{\mathrm{Min}}$ are, respectively, the largest and the smallest eigenvalues of $\hat{H}_{\text {ROT }}$. The expansion coefficients $a_{k}$ are determined by

$$
\begin{aligned}
a_{k}= & (-i)^{k} \exp \left(-i \Delta t \frac{1}{2}\left(E_{\mathrm{Max}}+E_{\mathrm{Min}}\right)\right)\left(2-\delta_{k, 0}\right) \\
& \times J_{k}\left(\Delta t \frac{1}{2}\left(E_{\mathrm{Max}}-E_{\mathrm{Min}}\right)\right)
\end{aligned}
$$

where $\delta_{k, 0}$ is the Kronecker symbol and $J_{k}(x)$ are the Bessel functions of the first kind.

\section{TIME-DEPENDENT MEAN-FIELD THEORY}

To solve the Schrödinger equation we seek a method which is simple to treat and, at the same time, becomes exact in the thermodynamic limit. Mean-field theory is believed to reproduce exactly the main features of the equilibrium quantum phase transition of the Dicke model for $j \rightarrow \infty$ [23], and therefore it should also yield good results for the dynamics in this limit. Mainly two methods have been used for establishing mean-field equations, the coherent-state representation [24], and the Holstein-Primakoff transformation [7]. These two approaches can be shown to be equivalent in the thermodynamic limit [25]. We adopt the coherent-state representation, using a procedure similar to the one presented in Ref. [26].

\section{A. Coherent-state representation}

We have seen that for the relevant operators $\hat{O}$ the expectation value $\left\langle\psi_{\mathrm{RD}}(t)|\hat{O}| \psi_{\mathrm{RD}}(t)\right\rangle$ with an evolution governed by the time-dependent Hamiltonian $\hat{H}_{\mathrm{RD}}(t)$ is simply related to $\left\langle\psi_{\text {ROT }}(t)|\hat{O}| \psi_{\text {ROT }}(t)\right\rangle$, with $\left|\psi_{\text {ROT }}(t)\right\rangle$ evolving according to the time-independent Hamiltonian $\hat{H}_{\text {ROT }}$, Eq. (16). For operators commuting with $\hat{J}_{z}$ the two expectation values are the same, while they are transformed to each other by the rotation, (19), for the operators $\hat{J}_{ \pm}$. In the following we calculate expectation values with respect to corotated states and transform the resulting expressions back to the unrotated frame at the end, if necessary. We use the notation

$$
\langle\hat{O}\rangle(t):=\left\langle\psi_{\mathrm{ROT}}(t)|\hat{O}| \psi_{\mathrm{ROT}}(t)\right\rangle .
$$

The Schrödinger equation, (15), leads to

$$
\partial_{t}\langle\hat{O}\rangle(t)=i\left\langle\left[\hat{H}_{\mathrm{ROT}}, \hat{O}\right]\right\rangle(t) .
$$

Specifically we find the equations of motion

$$
\begin{aligned}
\partial_{t}\left\langle\hat{J}_{z}\right\rangle(t) & =\frac{i \lambda}{\sqrt{2 j}}\left\langle\left(\hat{a}+\hat{a}^{\dagger}\right)\left(\hat{J}_{-}-\hat{J}_{+}\right)\right\rangle(t) \\
\partial_{t}\left\langle\hat{J}_{-}\right\rangle(t) & =-i\left(\omega_{0}+\delta_{\phi}\right)\left\langle\hat{J}_{-}\right\rangle(t)+i \sqrt{\frac{2}{j}} \lambda\left\langle\left(\hat{a}+\hat{a}^{\dagger}\right) \hat{J}_{z}\right\rangle(t) \\
\partial_{t}\langle\hat{a}\rangle(t) & =-i \omega\langle\hat{a}\rangle(t)-i \frac{\lambda}{\sqrt{2 j}}\left\langle\left(\hat{J}_{-}+\hat{J}_{+}\right)\right\rangle(t)
\end{aligned}
$$

Those for $\left\langle\hat{J}_{+}\right\rangle(t)$ and $\left\langle\hat{a}^{\dagger}\right\rangle(t)$ are obtained by complex conjugation.

Our main assumption is now that $\left|\psi_{\mathrm{ROT}}(t)\right\rangle$ is a coherent state, not only for $t=0$, but also for $t>0$. In the present context this means

$$
\left|\psi_{\mathrm{ROT}}(t)\right\rangle=|\alpha(t)\rangle \otimes|\zeta(t)\rangle=:|\alpha(t), \zeta(t)\rangle,
$$

with spin-coherent states

$$
|\zeta(t)\rangle=\left(1+|\zeta(t)|^{2}\right)^{-j} e^{\zeta(t) \hat{J}_{+}}|j,-j\rangle
$$

and bosonic coherent states

$$
|\alpha(t)\rangle=e^{-\frac{1}{2}|\alpha(t)|^{2}} e^{\alpha(t) a^{\dagger}}|0\rangle .
$$


The expectation values of the relevant operators with respect to the coherent state, (26), are

$$
\begin{aligned}
\left\langle\hat{J}_{+}\right\rangle(t) & =2 j \frac{\zeta^{*}(t)}{1+|\zeta(t)|^{2}}=\left\langle\hat{J}_{-}\right\rangle^{*}(t), \\
\left\langle\hat{J}_{z}\right\rangle(t) & =-j \frac{1-|\zeta(t)|^{2}}{1+|\zeta(t)|^{2}}, \\
\langle\hat{a}\rangle(t) & =\alpha(t), \quad\left\langle\hat{a}^{\dagger} \hat{a}\right\rangle(t)=|\alpha(t)|^{2} .
\end{aligned}
$$

We note that if $\left|\psi_{\mathrm{ROT}}(t)\right\rangle$ is a coherent state, then this is also true for $\left|\psi_{\mathrm{RD}}(t)\right\rangle$, which is related to $\left|\psi_{\mathrm{ROT}}(t)\right\rangle$ by Eq. (14), and vice versa. To see this, one verifies that the application of $\hat{R}_{z}^{\dagger}(t)$ on $|\zeta(t)\rangle$ produces a spin-coherent state $\left|\zeta_{\mathrm{RD}}(t)\right\rangle$ in the original frame, with

$$
\zeta_{\mathrm{RD}}(t)=e^{i \phi(t)} \zeta(t)
$$

\section{B. Classical dynamical system}

The equations of motion, (25), can now be linked to a classical dynamical system of two coupled oscillators using the parametrization

$$
\begin{aligned}
\zeta(t) & =\frac{1}{\sqrt{4-\left[Q^{2}(t)+P^{2}(t)\right]}}[Q(t)+i P(t)], \\
\alpha(t) & =\sqrt{\frac{j}{2}}[q(t)+i p(t)] .
\end{aligned}
$$

Inserting these relations into Eqs. (25) and isolating real and imaginary parts we find the equations of motion

$$
\begin{aligned}
& \dot{Q}=\Omega P-\lambda q \frac{Q P}{\sqrt{4-\left(Q^{2}+P^{2}\right)}}, \\
& \dot{P}=-\Omega Q+\lambda q \frac{2 Q^{2}+P^{2}-4}{\sqrt{4-\left(Q^{2}+P^{2}\right)}}, \\
& \dot{q}=\omega p, \\
& \dot{p}=-\omega q-\lambda Q \sqrt{4-\left(Q^{2}+P^{2}\right)},
\end{aligned}
$$

where $\Omega$ is the renormalized level splitting, (17). With the same parametrization the expectation value of $\hat{H}_{\text {ROT }}$ yields a classical Hamiltonian, $H_{\mathrm{cl}}:=\Omega+\frac{1}{j}\left\langle\alpha(t), \zeta(t)\left|\hat{H}_{\mathrm{ROT}}\right| \alpha(t), \zeta(t)\right\rangle$; explicitly,

$$
H_{\mathrm{cl}}=\frac{\Omega}{2}\left(Q^{2}+P^{2}\right)+\frac{\omega}{2}\left(q^{2}+p^{2}\right)+\lambda Q q \sqrt{4-\left(Q^{2}+P^{2}\right)} .
$$

Similarly, using Eq. (29) we find for the mean photon number, (11),

$$
n_{\mathrm{ph}}=\frac{1}{j}|\alpha|^{2}=\frac{1}{2}\left(q^{2}+p^{2}\right),
$$

while the atomic inversion, (12), is found to be

$$
n_{\mathrm{at}}=\frac{2|\zeta|^{2}}{1+|\zeta|^{2}}=\frac{1}{2}\left(Q^{2}+P^{2}\right)
$$

One readily verifies that Eqs. (32) are the canonical equations for the Hamiltonian, (33), and therefore $Q, P, q$, and $p$ are the canonical coordinates of a dynamical system of 2 degrees of freedom. Once Eqs. (32) have been solved it is easy to find the corresponding coordinates in the original system using Eq. (30). The photonic variables $q$ and $p$ remain the same, while the atomic variables $Q$ and $P$ become

$$
\begin{aligned}
Q_{\mathrm{RD}}(t) & =Q(t) \cos \phi(t)-P(t) \sin \phi(t), \\
P_{\mathrm{RD}}(t) & =Q(t) \sin \phi(t)+P(t) \cos \phi(t)
\end{aligned}
$$

in the unrotated frame.

We have verified that the same classical Hamiltonian emerges either when applying the Holstein-Primakoff transformation from spins to bosons or when using the saddle-point approximation of the coherent-state path integral [24].

\section{Stationary states and linear modes}

We show now that the stationary solutions of the classical equations of motion reproduce the phases characterizing the ground state of $\hat{H}_{\text {ROT }}$ in the limit $j \rightarrow \infty$. The arguments are similar to those used in Ref. [7] for the time-independent Dicke Hamiltonian. Inserting $\dot{Q}=\dot{P}=\dot{q}=\dot{p}=0$ into Eqs. (32) we find a first obvious solution $Q_{0}=P_{0}=q_{0}=p_{0}=0$ for $\lambda<$ $\lambda_{c}=\frac{1}{2} \sqrt{\omega \Omega}$. It describes the normal phase with vanishing photon density. The linearized equations of motion around this point are

$$
\begin{aligned}
& \dot{Q}=\Omega P, \quad \dot{P}=-\Omega Q-2 \lambda q, \\
& \dot{q}=\omega p, \quad \dot{p}=-\omega q-2 \lambda Q,
\end{aligned}
$$

and the eigenmode frequencies are given by

$$
\varepsilon_{ \pm}^{2}=\frac{1}{2}\left\{\Omega^{2}+\omega^{2} \pm \sqrt{\left(\Omega^{2}-\omega^{2}\right)^{2}+16 \lambda^{2} \Omega \omega}\right\} .
$$

$\varepsilon_{-}$tends to 0 for $\lambda \rightarrow \lambda_{c}$ and thus represents the soft mode of the transition.

Two stationary solutions are found for $\lambda>\lambda_{c}$, namely,

$$
\begin{aligned}
Q_{0} & = \pm \sqrt{2}\left(1-\frac{\lambda_{c}{ }^{2}}{\lambda^{2}}\right)^{\frac{1}{2}}, \\
q_{0} & =\mp \frac{2 \lambda}{\omega}\left(1-\frac{\lambda_{c}{ }^{4}}{\lambda^{4}}\right)^{\frac{1}{2}}, \quad P_{0}=p_{0}=0 .
\end{aligned}
$$

They describe the superradiant phase with densities

$$
\begin{aligned}
& n_{\mathrm{ph}}=\frac{1}{j}|\alpha|^{2}=\frac{2 \lambda^{2}}{\omega^{2}}\left(1-\frac{\lambda_{c}{ }^{4}}{\lambda^{4}}\right), \\
& n_{\mathrm{at}}=\frac{2|\zeta|^{2}}{1+|\zeta|^{2}}=1-\frac{\lambda_{c}{ }^{2}}{\lambda^{2}} .
\end{aligned}
$$

We consider small deviations $X=Q-Q_{0}, x=q-q_{0}$, $P$, and $p$ from the stationary solutions, (39), of the superradiant phase. To linear order in these coordinates the equations of motion, (32), read

$$
\begin{aligned}
& \dot{X}=\frac{2}{\omega}\left(\lambda^{2}+\lambda_{c}{ }^{2}\right) P, \\
& \dot{P}=-\frac{\sqrt{8} \lambda_{c}{ }^{2}}{\sqrt{\lambda^{2}+\lambda_{c}^{2}}} x-\frac{8 \lambda^{4}}{\omega\left(\lambda^{2}+\lambda_{c}{ }^{2}\right)} X, \\
& \dot{x}=\omega p, \quad \dot{p}=-\omega x-\frac{\sqrt{8} \lambda_{c}{ }^{2}}{\sqrt{\lambda^{2}+\lambda_{c}^{2}}} X .
\end{aligned}
$$


The eigenmode frequencies of this linear system are

$$
\varepsilon_{ \pm}^{2}=\frac{1}{2}\left\{\omega^{2}+\frac{16 \lambda^{4}}{\omega^{2}} \pm \sqrt{\left(\omega^{2}-\frac{16 \lambda^{4}}{\omega^{2}}\right)^{2}+4(\omega \Omega)^{2}}\right\} .
$$

The solution with eigenfrequency $\varepsilon_{-}$again represents a soft mode, $\varepsilon_{-} \rightarrow 0$ for $\lambda \rightarrow \lambda_{c}$. Equations (38), (39), and (42) agree with the corresponding expressions for the undriven Dicke model in Ref. [7], where the Holstein-Primakoff transformation has been used.

This stability analysis shows that in mean-field approximation the rotation applied to the Dicke Hamiltonian changes the phase diagram. The critical point $\lambda_{c}^{0}=\sqrt{\omega \omega_{0}} / 2$ is shifted to $\lambda_{c}=\sqrt{\omega \Omega} / 2$, where $\Omega=\omega_{0}+\delta_{\phi}$. The expressions for the photon density and the atomic inversion remain the same as in the time-independent Dicke model, except that $\lambda_{c}^{0}$ is replaced by $\lambda_{c}$. Also, the eigenmode frequencies of the time-dependent Dicke Hamiltonian are simply obtained from those of the time-independent Hamiltonian by replacing $\omega_{0}$ with $\Omega$.

The stationary points for the corotating frame are readily transformed to the original frame using Eq. (36). The stationary point of the normal phase does not change, but that of the superradiant phase is transformed into uniform circular motion in the $Q_{\mathrm{RD}}-P_{\mathrm{RD}}$ plane with radius $2 \sqrt{n_{\mathrm{at}}}$ and angular velocity $\delta_{\phi}$.

For an initial condition which is close to a stationary point (in the corotated frame) the evolution of the system is governed by the linearized equations of motion and will in general be a superposition of harmonic oscillations with frequencies $\varepsilon_{ \pm}$. The trajectories form complicated Lissajous patterns, as exemplified in Fig. 2. The trajectory [thin (purple) line] starting close to the stationary circle [thick (blue) line] shows that, in addition to the circular motion of angular frequency $\delta_{\phi}$, there is a transverse harmonic oscillation with a frequency $\epsilon_{-} \approx 1-\frac{1}{8 \lambda^{4}}=0.875$ (see Appendix A).

\section{DYNAMICALLY GENERATED DARKNESS}

We discuss now the evolution after the rotation, (8), is switched on suddenly at $t=0$. Thus the system is supposed to be in the ground state $\left|\psi_{0}\right\rangle$ of the time-independent Dicke Hamiltonian $\hat{H}_{\mathrm{D}}$ for $t<0$ and to evolve according to the time-dependent Dicke Hamiltonian $\hat{H}_{\mathrm{RD}}(t)$ for $t>0$. In the rotated frame we have to solve the Schrödinger equation, (15), for $\left|\psi_{\text {ROT }}(t)\right\rangle$ with the initial condition $\left|\psi_{\text {ROT }}(0)\right\rangle=\left|\psi_{0}\right\rangle$. We have used both the numerical technique for the full quantum problem (explained in Sec. III C) and the mean-field approximation (described in Sec. IV) for calculating important quantities such as the photon number and the atomic inversion.

Nothing special will happen for $\lambda<\lambda_{c}^{0}$, where the system starts in the normal phase of $\hat{H}_{\mathrm{D}}$ for $t=0$ and proceeds in the normal phase of $\hat{H}_{\mathrm{RD}}(t)$ for $t>0$. Therefore we concentrate on couplings $\lambda>\lambda_{c}^{0}$. We use preferentially the parameter values $\omega=\omega_{0}=\delta_{\phi}=1$, for which the critical points are $\lambda_{c}^{0}=0.5$ and $\lambda_{c}=1 / \sqrt{2} \approx 0.707$. For the results presented here we have chosen as the initial state the mean-field ground state of $\hat{H}_{\mathrm{D}}$, both for the numerical evaluation of the exact quantum dynamics and for the treatment of the classical dynamical system. We have verified that starting with the exact ground

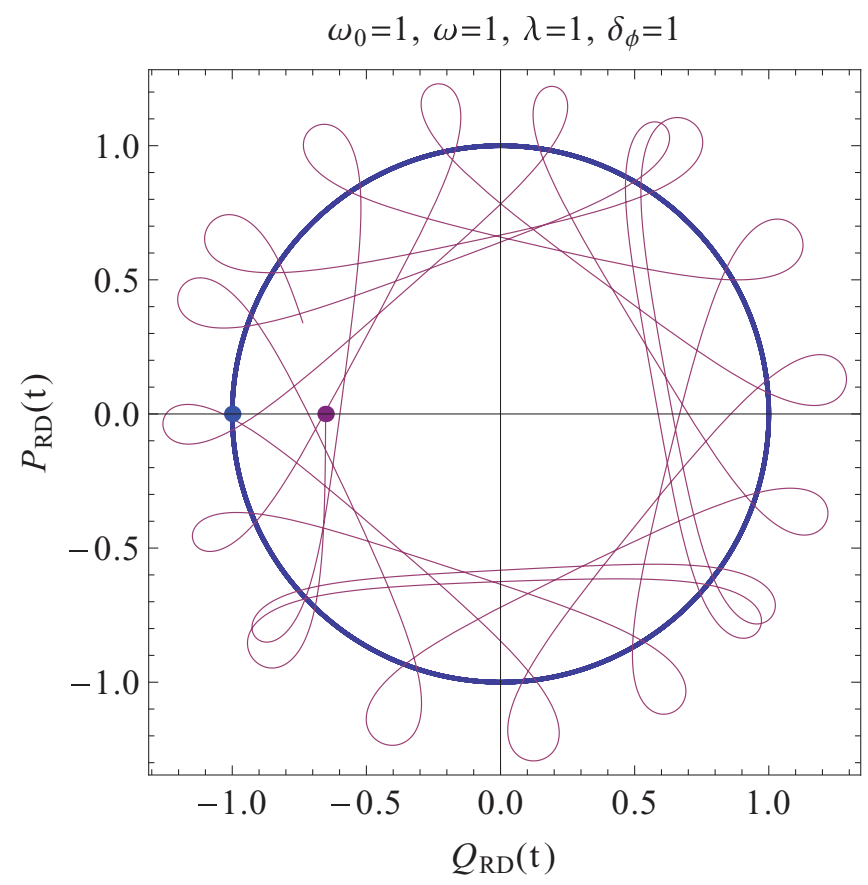

FIG. 2. (Color online) Phase portrait $\left[Q_{\mathrm{RD}}(t), P_{\mathrm{RD}}(t)\right]$ : The thick (blue) circle represents the stationary solution in the corotating frame, corresponding to the initial condition $Q_{\mathrm{RD}}(0)=Q_{0}, P_{\mathrm{RD}}(0)=$ $0, q(0)=q_{0}$, and $p(0)=0$. The trajectory [thin (purple) line] has the initial condition slightly away from the stationary point, i.e., $Q_{\mathrm{RD}}(0)=Q_{0}+0.347851, P_{\mathrm{RD}}(0)=0, q(0)=q_{0}$, and $p(0)=0$.

state of $\hat{H}_{\mathrm{D}}$ (for the numerical procedure) does not modify the main results. The initial values of the classical coordinates $Q(0), q(0), P(0)$, and $p(0)$ are given by Eqs. (39), with $\lambda_{c}$ replaced by $\lambda_{c}^{0}$. In view of Eqs. (31) the corresponding parameters of the initial coherent state are

$$
\begin{aligned}
& \alpha(0)=\sqrt{\frac{j}{2}} q(0)=\mp \frac{\sqrt{2 j}}{\omega \lambda} \sqrt{\lambda^{4}-\left[\lambda_{c}^{0}\right]^{4}}, \\
& \zeta(0)=\frac{Q(0)}{\sqrt{4-Q^{2}(0)}}= \pm \sqrt{\frac{\lambda^{2}-\left[\lambda_{c}^{0}\right]^{2}}{\lambda^{2}+\left[\lambda_{c}^{0}\right]^{2}}} .
\end{aligned}
$$

Figure 3 shows results for the mean photon number for three couplings. Deep in the superradiant phase of $\hat{H}_{\mathrm{RD}}(\lambda=1.3)$ a simple oscillatory behavior is observed, for both mean-field and full quantum solutions, with $j=10$ in the latter case. This behavior is readily understood in the linearized limit of the mean-field equations, where the coordinates execute harmonic motions, with frequencies $\varepsilon_{ \pm}$, as explained in Appendix A. For $\lambda \gg \lambda_{c}$ the main contribution comes from the mode with frequency $\varepsilon_{-} \approx 1-1 / 8 \lambda^{4}$, in perfect agreement with Fig. 3. The results for $\lambda=1$ are still reproduced rather well by the linearized dynamics, but not for $\lambda=0.6$, where the anharmonic part of the potential becomes important for the chosen initial conditions. For this coupling the (nonlinear) mean-field behavior agrees only initially with the dynamics obtained from the numerical integration of the Schrödinger equation, but not at later times, where the quantum evolution appears to reach a constant value. We attribute this difference 

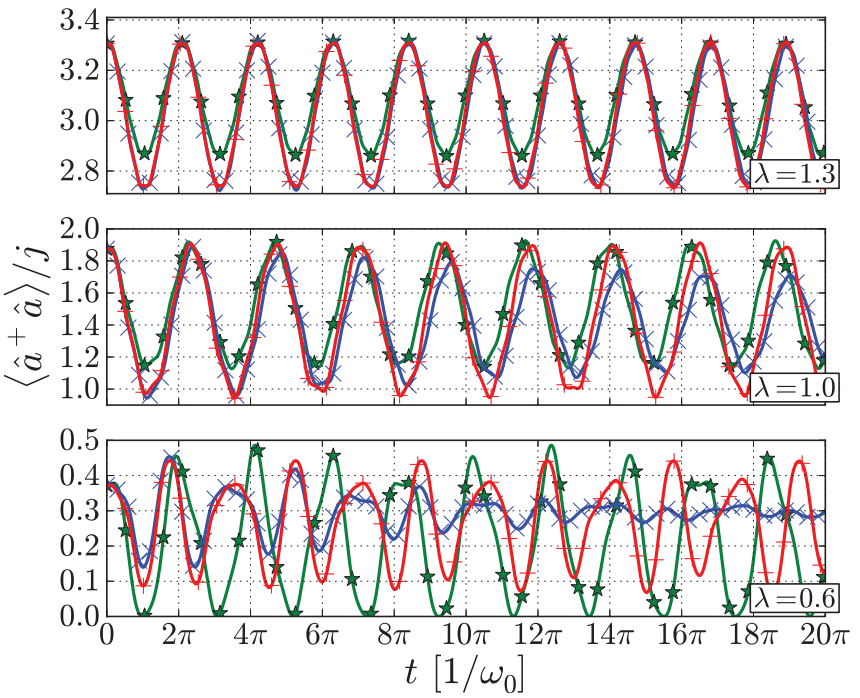

FIG. 3. (Color online) Time dependence of the mean photon number at resonance $\left(\omega=\omega_{0}=1.0\right)$ and for a driving velocity $\delta_{\phi}=1.0$. The (red) curves with crosses represent the time evolution of the nonlinear mean-field equations, the (green) curves with asterisks show the linearized limit of these equations, and the (blue) curves with $X$ 's have been obtained by numerical integration of the Schrödinger equation for a finite system $\left(j=10, n_{\mathrm{M}}=60\right)$. The first two cases (couplings $\lambda=1.3,1$ ) correspond to the superradiant phase of the time-dependent Dicke model; the third $(\lambda=0.6)$, to its normal phase. All three couplings exceed the critical value of the time-independent Dicke model $\left(\lambda_{c}^{0}=0.5\right)$.

to decoherence in the quantum case, for which, at times longer than those shown in the Fig. 3, the initial pattern is restored. Mean-field theory remains, by construction, a coherent state.

We discuss now the mean photon number $n_{\mathrm{ph}}(t)$. If the system evolves according to $\hat{H}_{\mathrm{D}}$ and if the initial state $\left|\psi_{\mathrm{RD}}(0)\right\rangle$ is the ground state of $\hat{H}_{\mathrm{D}}$, then $n_{\mathrm{ph}}$ does not depend on time and is just the order parameter of the time-independent Dicke model, except that in the mean-field expression, Eq. (40), $\lambda_{c}$ is replaced by $\lambda_{c}^{0}$. An analogous statement holds if the evolution is governed by $\hat{H}_{\mathrm{RD}}$ with the ground state of $\hat{H}_{\mathrm{ROT}}$ as the initial state; then $n_{\mathrm{ph}}$ is given by Eq. (40). The two cases are illustrated as dashed lines in Fig. 4. A qualitatively different behavior is found for the scenario discussed above, where the evolution is governed by $\hat{H}_{\mathrm{RD}}$, but the initial state is the ground state of $\hat{H}_{\mathrm{D}}$. The solid lines in Fig. 4 show $n_{\mathrm{ph}}$ after a single period of rotation $T_{\phi}:=2 \pi / \delta_{\phi}$. For both $\lambda_{c}^{0}<\lambda<$ $\lambda_{c}$ and $\lambda \gg \lambda_{c}$ the mean photon number $n_{\mathrm{ph}}\left(T_{\phi}\right)$ does not differ much from its initial value. For $\lambda$ slightly larger than $\lambda_{c}, n_{\mathrm{ph}}\left(T_{\phi}\right)$ is strongly reduced compared to its value at $t=0$. This remarkable dynamically generated darkness is found both in the mean-field approximation and in the quantum evolution for finite $j$.

The time-averaged mean photon number

$$
\bar{n}_{\mathrm{ph}}:=\frac{1}{j T} \int_{0}^{T} d t\left\langle\psi(t)\left|\hat{a}^{\dagger} \hat{a}\right| \psi(t)\right\rangle
$$

is shown in Fig. 5 for $T \gg T_{\phi}$. In contrast to $n_{\mathrm{ph}}\left(T_{\phi}\right), \bar{n}_{\mathrm{ph}}$ approaches the steady-state value of $\hat{H}_{\mathrm{RD}}$ for $\lambda \gg \lambda_{c}$ (and not that of $\left.\hat{H}_{\mathrm{D}}\right)$. This is readily understood in the linearized

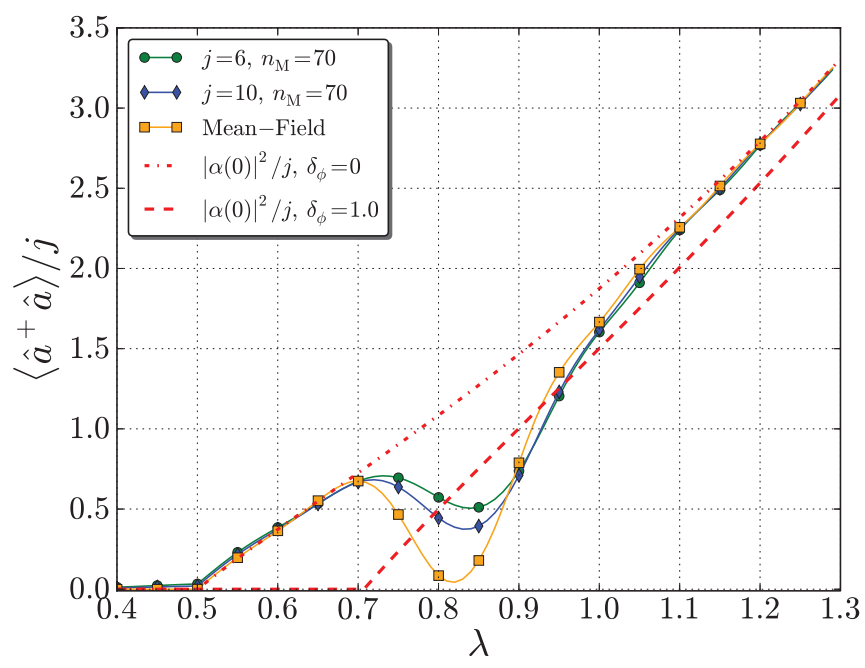

FIG. 4. (Color online) Mean photon number as a function of the coupling strength after a single period of revolution $T_{\phi}$ and for $\omega=$ $\omega_{0}=\delta_{\phi}=1$. Dashed lines represent the ground-state values for $\hat{H}_{\mathrm{D}}$ and $\hat{H}_{\text {ROT }}$, respectively. Solid lines were obtained starting with the ground state of $\hat{H}_{\mathrm{D}}$ at $t=0$, but evolving with $\hat{H}_{\mathrm{RD}}$ for $0<t<T_{\phi}$, both in the mean-field approximation and by numerically solving the Schrödinger equation for finite $j$.

limit of the classical dynamics, where $q(t), p(t)$ oscillate with frequency $\varepsilon_{-} \approx \omega$ about the stationary values $q_{0}$ and $p_{0}$, as shown in Appendix A. This implies that for $\omega=\delta_{\phi}=1$ the coordinates and thus also $n_{\mathrm{ph}}$ recover their original values after a time $T_{\phi}$, in agreement with Fig. 4 , but the time-averaged mean photon number $\bar{n}_{\mathrm{ph}}$ is close to its stationary value, as in Fig. 5.

Figure 5 also shows that the reduction in the photon number not only appears at $T_{\phi}$, but also is seen after taking the time average. A clear minimum is found if the time-averaged photon

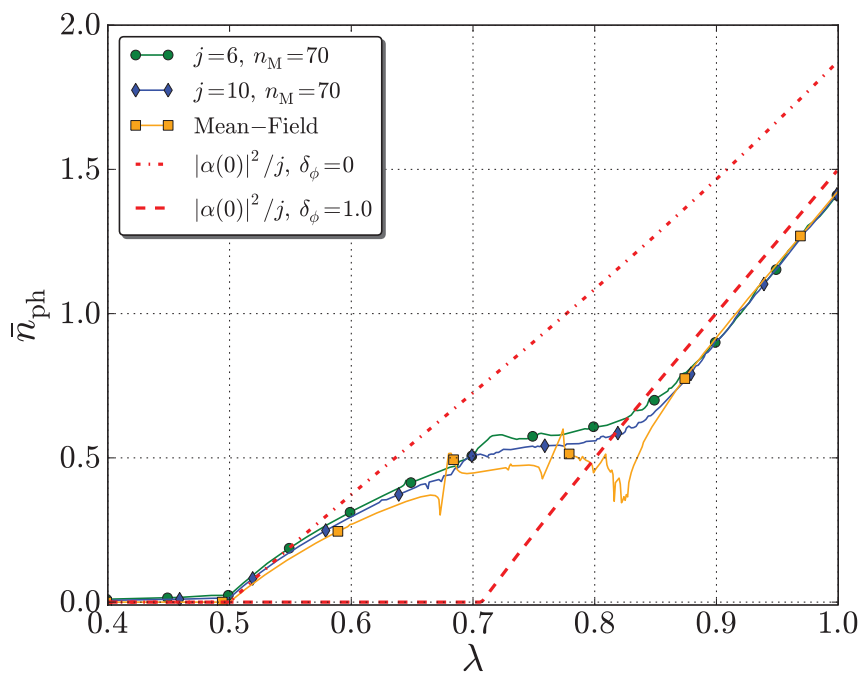

FIG. 5. (Color online) Coupling dependence of the mean photon number averaged over a time $T=150 T_{\phi}$ for parameter values $\omega=$ $\omega_{0}=\delta_{\phi}=1$. Dashed lines represent the ground-state values for $\hat{H}_{\mathrm{D}}$ and $\hat{H}_{\text {ROT }}$, respectively. The (green) line with circles was obtained by numerically solving the Schrödinger equation for $j=6$ and the (blue) line with diamonds for $j=10$, with cutoff $n_{\mathrm{M}}=70$ in both cases. 


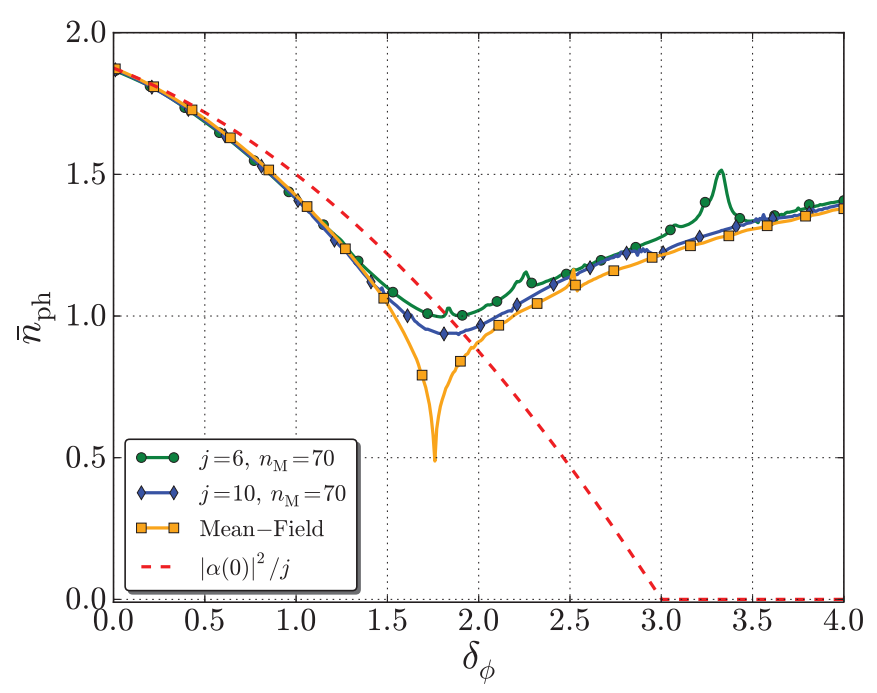

FIG. 6. (Color online) Time-averaged mean photon number (solid lines) compared to the initial value (dashed line) as a function of the rotation velocity $\delta_{\phi}$ for $\lambda=\omega=\omega_{0}=1$ and $T=150 T_{\phi}$. There is a clear minimum, which is very sharp in the mean-field approximation [(yellow) line with squares] and more shallow when calculated numerically for the quantum case with $j=6$ [(green) line with circles] and $j=10$ [(blue) line with diamonds].

number is plotted as a function of the driving velocity $\delta_{\phi}$ for a fixed coupling strength. This is shown in Fig. 6, where the darkening effect is particularly pronounced in the mean-field approximation.

To illustrate the phenomenon of dynamically generated darkness we have studied a simple classical system of a particle moving on a plane in an isotropic potential $V(|\mathbf{x}|)$ with a maximum $V_{0}$ at the origin and a minimum at $|\mathbf{x}|=\rho_{0}$ (Mexican hat). Details are described in Appendix B. For a vanishing initial momentum $\mathbf{p}(0)$ and finite $\mathbf{x}(0)$ the particle exhibits radial oscillations around $\rho_{0}$ if the energy $E$ is lower than $V_{0}$; for $E>V_{0}$ it passes over the potential hump at the center and moves back and forth between the turning points $\pm \mathbf{x}_{\text {max }}$. For $E \approx V_{0}$ the motion is slowed down close to the center, where the particle spends most of the time. The time average of both $\mathbf{x}^{2}(t)$ and $\mathbf{p}^{2}(t)$ tends to 0 for $E \rightarrow V_{0}$.

We use now the result found in the case of the Mexican hat to estimate the critical coupling for maximal slowing-down in the case of the time-dependent Dicke model. We expect this to occur if the energy for the given initial conditions is equal to the maximum of the potential at the origin; i.e., $H_{\mathrm{cl}}$, given by Eq. (33), vanishes for the coordinates of the stationary state of $\hat{H}_{\mathrm{D}}$. We find the following estimate for this dynamic critical point:

$$
\lambda_{c}^{(\mathrm{dyn})} \approx \frac{1}{2} \sqrt{\omega\left(\omega_{0}+2 \delta_{\phi}\right)}
$$

This represents a rough guess because the potential in the case of the Dicke model is both anisotropic and velocity dependent. Therefore it is not surprising that expression (45) does not reproduce exactly the dynamic critical line found by solving the mean-field equations and shown in Fig. 7. Rather the numerical data are well fitted by a power law, $\lambda_{c}^{(\mathrm{dyn})}=\lambda_{c}^{0}+0.327 \delta_{\phi}^{3 / 4}$.

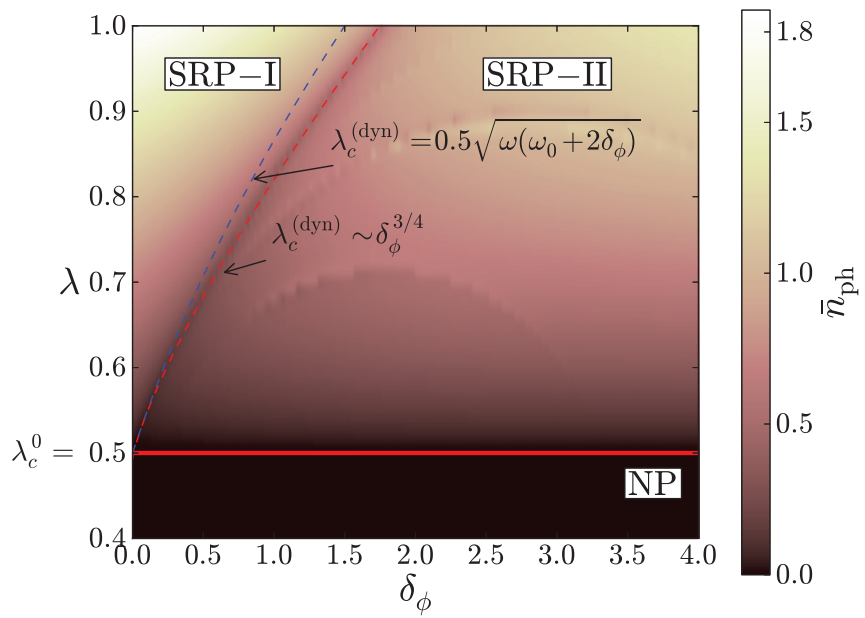

FIG. 7. (Color online) Time-averaged mean photon number as a function of both driving velocity $\delta_{\phi}$ and coupling strength $\lambda$, according to mean-field theory. We chose parameter values $\omega=\omega_{0}=1.0$ and averaged over a time $T=150 T_{\phi}$. The leftward (blue) dashed line corresponds to the theoretical estimate of Eq. (45); the rightward (red) dashed line is the fit to the observed minimum.

To exemplify the complicated dynamics of the timedependent Dicke model we present in Fig. 8 a few trajectories, as obtained by solving numerically the equations of motion, (32). The stationary states of the time-independent Dicke model have again been chosen as initial conditions. The contour lines in Fig. 8 represent the static limit $V_{\mathrm{cl}}(Q, q, 0)$
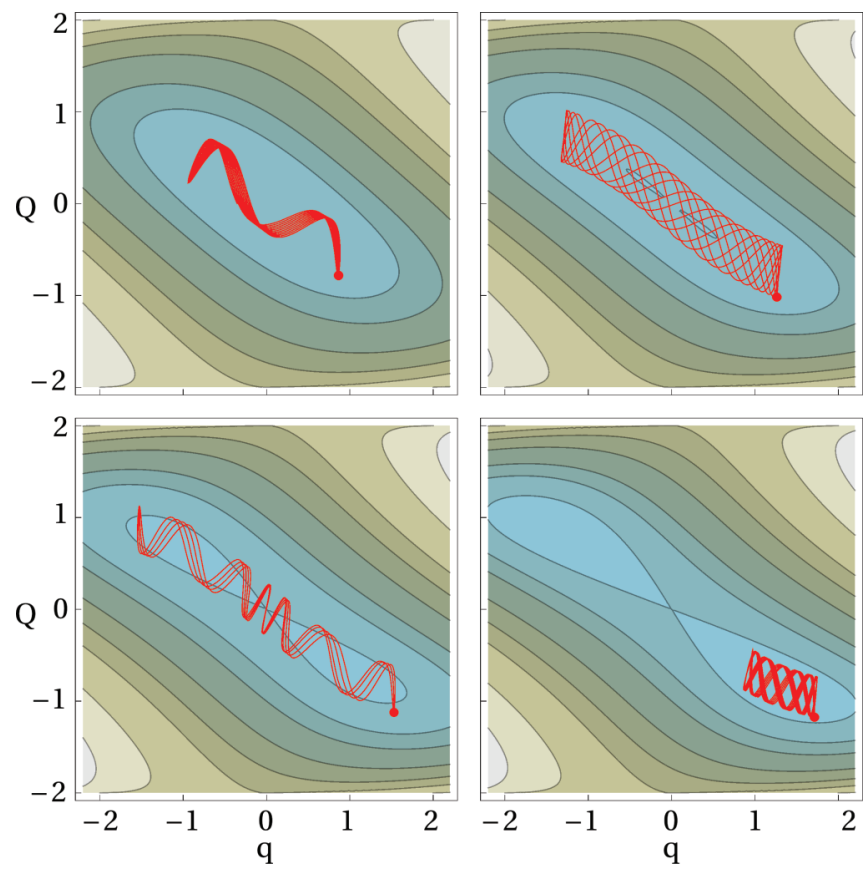

FIG. 8. (Color online) Contours of the potential $V_{\mathrm{cl}}(Q, q, 0)$, with the trajectories $(Q, q)$ illustrated by bold central (red) curves. The filled (red) circles at the bottom of these curves mark the initial states given by Eqs. (39) with $\lambda_{c}$ replaced by $\lambda_{c}^{0}$. The four trajectories have been calculated for fixed parameter values, $\omega=\omega_{0}=\delta_{\phi}=1$, but different couplings, $\lambda=0.6,0.72,0.823$, and 0.9 (from left to right and top to bottom). 
of the classical potential

$$
V_{\mathrm{cl}}(Q, q, P)=\frac{\Omega}{2} Q^{2}+\frac{\omega}{2} q^{2}+\lambda Q q \sqrt{4-\left(Q^{2}+P^{2}\right)} .
$$

The first trajectory is representative for the range $\lambda_{c}^{0}<$ $\lambda<\lambda_{c}$, where the motion is rather regular, while the fourth trajectory illustrates the strong-coupling region $\lambda \gg \lambda_{c}$, where the motion is limited to the region around one potential minimum. This harmonic motion is correctly reproduced by the linearized limit of the equation of motion. The second trajectory shows the chaotic motion slightly above the critical point $\left(\lambda=\lambda_{c}\right)$. The third example corresponds to the coupling constant, for which $\bar{n}_{\mathrm{ph}}$ has a pronounced minimum according to the mean-field theory ( $\lambda=0.823$; see Fig. 5). In this case the trajectory is seen to have inversion symmetry, thus it passes through the origin. However, due to the transverse oscillations the motion never stops and therefore the averaged mean photon number is only reduced and does not tend to 0 .

In the scenario considered above the system is prepared in the ground state of the time-independent Dicke Hamiltonian, then suddenly the rotation is switched on and drives the system out of equilibrium. In that case we have found a reduced photon number but not a complete darkening. We address now the question whether it is possible to fine-tune the initial conditions in such a way that the dynamically generated darkness is complete, i.e., $\bar{n}_{\mathrm{ph}}=0$. To achieve this we simply start at the origin of the coordinate system and also assume that one of the initial momenta vanishes, while the other is infinitesimally small. We then follow the evolution for $\lambda=0.823$, the coupling where the previous protocol has provided a minimum in $\bar{n}_{\mathrm{ph}}$. The system remains close to the initial point in phase space for a long time until it moves away and acquires rather large values of both spatial coordinates and momenta. Such a point can then be used as the initial condition for other values of $\lambda$. Figure 9 shows both the mean photon number $\bar{n}_{\mathrm{ph}}$ and the atomic inversion $\bar{n}_{\text {at }}$ as a function of $\lambda$ for the fine-tuned starting point $Q(0)=0.9049, P(0)=-1.6382$, $q(0)=-0.0204$, and $p(0)=0.171581$. As expected, both $\bar{n}_{\mathrm{ph}}$ and $\bar{n}_{\text {at }}$ approach 0 for $\lambda=0.823$, or, stated otherwise, there is a complete slowing-down at the origin, as in the case of the Mexican hat. Other minima of $\bar{n}_{\mathrm{ph}}$ occur at $\lambda=0.693$ and $\lambda=0.507$, but $\bar{n}_{\text {at }}$ shows the opposite behavior at the latter point; it exhibits a maximum instead of a minimum. For strong couplings both $\bar{n}_{\mathrm{ph}}$ and $\bar{n}_{\mathrm{at}}$ approach the stationary values, as predicted by the solutions of the linearized mean-field equations.

\section{INTERPLAY OF GEOMETRIC AND DYNAMIC PHASES}

In the previous section we have interpreted the dynamically generated darkness in the framework of time-dependent meanfield theory and attributed the effect to a slowing-down of the classical motion. For a system with a small number of two-level atoms mean-field theory is no longer valid and one has to solve the time-dependent Schrödinger equation. We believe that in this case the dynamically generated darkness can be traced back to an interplay between geometric and dynamic phases appearing away from equilibrium [27]. To support this claim

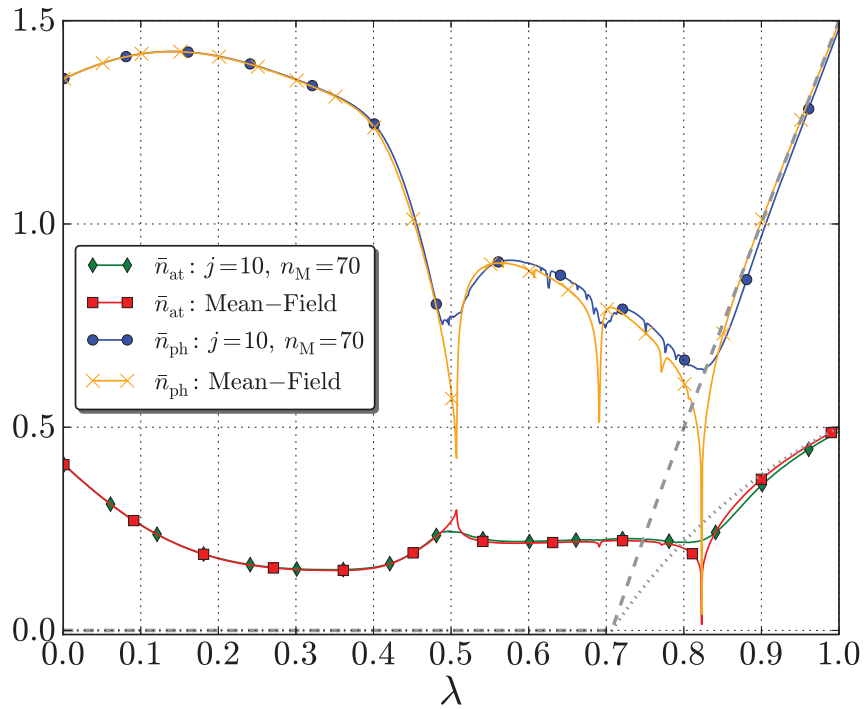

FIG. 9. (Color online) Coupling dependence of the mean photon number and the atomic inversion averaged over a time $T=150 T_{\phi}$ for $\omega=\omega_{0}=\delta_{\phi}=1$. Dashed lines represent the ground-state values for $\hat{H}_{\text {ROT }}$. The (blue) line with circles, $\bar{n}_{\mathrm{ph}}$, and (green) line with diamonds, $\bar{n}_{\text {at }}$, have been calculated by numerically solving the Schrödinger equation for $j=10$ and $n_{\mathrm{M}}=70$. The mean-field results, the (orange) line with crosses for $\bar{n}_{\mathrm{ph}}$ and the red line with squares for $\bar{n}_{\text {at }}$, exhibit sharp features and drop steeply to 0 for $\lambda=0.823$. The system is initially in the fine-tuned coherent state with $Q(0)=0.9049, P(0)=-1.6382, q(0)=-0.0204$, and $p(0)=0.171581$.

we represent the time-dependent Schrödinger equation

$$
i \partial_{t}\left|\psi_{\mathrm{RD}}(t)\right\rangle=\hat{H}_{\mathrm{RD}}(t)\left|\psi_{\mathrm{RD}}(t)\right\rangle
$$

in the instantaneous basis $\left|\varphi_{l}(t)\right\rangle$ of $\hat{H}_{\mathrm{RD}}(t)$, defined by

$$
\hat{H}_{\mathrm{RD}}(t)\left|\varphi_{l}(t)\right\rangle=\epsilon_{l}(t)\left|\varphi_{l}(t)\right\rangle,
$$

with $l$ enumerating all the eigenstates of $\hat{H}_{\mathrm{RD}}(t)$ at an instant $t$; e.g., $\left|\varphi_{1}(t)\right\rangle$ is the ground state of $\hat{H}_{\mathrm{RD}}(t)$ at time $t$. The time evolution of the instantaneous eigenstates of $\hat{H}_{\mathrm{RD}}(t)$ is simply given by a rotation of the eigenstates of $\hat{H}_{\mathrm{D}},\left|\varphi_{l}(t)\right\rangle=$ $\hat{R}_{z}^{\dagger}(t)\left|\varphi_{l}(0)\right\rangle$, with time-independent eigenvalues $\epsilon_{l}(t)=\epsilon_{l}(0)$ (those of $\hat{H}_{\mathrm{D}}$ ), because the rotationally driven Dicke Hamiltonian $\hat{H}_{\mathrm{RD}}(t)$ is obtained by applying the unitary transformation, (8), to the time-independent Dicke Hamiltonian $\hat{H}_{\mathrm{D}}$. Inserting the expansion $\left|\psi_{\mathrm{RD}}(t)\right\rangle=\sum_{l} c_{l}(t)\left|\varphi_{l}(t)\right\rangle$ into the Schrödinger equation yields a differential equation for the coefficients $c_{l}(t)$,

$$
\frac{d c_{l}}{d t}=i\left(-\epsilon_{l}+A_{l l}\right) c_{l}+i \sum_{k, k \neq l} A_{l k} c_{k}
$$

where we introduced the notation $A_{l k}(t):=i\left\langle\varphi_{l}(t)\left|\frac{d}{d t}\right| \varphi_{k}(t)\right\rangle$. The integral of the so-called Berry connection $A_{l l}(t)=$ $i\left\langle\varphi_{l}(t)\left|\frac{d}{d t}\right| \varphi_{l}(t)\right\rangle[28]$ is the geometric phase of the $l$ th instantaneous eigenstate, the Berry phase [29],

$$
\gamma_{l}(t)=\int_{0}^{t} d \tau i\left\langle\varphi_{l}(\tau)\left|\frac{d}{d \tau}\right| \varphi_{l}(\tau)\right\rangle .
$$

Equation (49) shows that for an adiabatic evolution (where transitions to different levels $k \neq l$ can be neglected) each 
instantaneous eigenstate $\left|\varphi_{l}(t)\right\rangle$ acquires not only the wellknown dynamic phase $\mathcal{E}_{l}(t)=\int_{0}^{t} d \tau \epsilon_{l}$ but also a geometric phase, $\gamma_{l}(t)=\int_{0}^{t} d \tau A_{l l}(\tau)$. Applying the gauge transformation

$$
c_{l}(t)=\chi_{l}(t) \exp \left(i \int_{0}^{t} d \tau\left[-\epsilon_{l}+A_{l l}(\tau)\right]\right)
$$

to Eq. (49), we obtain the equation

$$
\frac{d \chi_{l}}{d t}=i \sum_{k, k \neq l} A_{l k}(\phi) \exp \left(i \mathcal{E}_{l k}(t)-i \gamma_{l k}(t)\right) \chi_{k}(t),
$$

which emphasizes the interplay between the dynamic phase $\mathcal{E}_{l k}(t)=\mathcal{E}_{l}(t)-\mathcal{E}_{k}(t)$ and the geometric phase $\gamma_{l k}(t)=\gamma_{l}(t)-$ $\gamma_{k}(t)$. For our model with a constant rotation speed, $\phi(t)=\delta_{\phi} t$, the characteristic time scale of the drive is $T_{\phi}=2 \pi / \delta_{\phi}$. After a single rotation the dynamic phase is given by $\mathcal{E}_{l k}(t)=$ $2 \pi\left(\epsilon_{l}-\epsilon_{k}\right) / \delta_{\phi}$ and is large for $\epsilon_{l} \neq \epsilon_{k}$ if $\delta_{\phi}$ is small. Thus for adiabatic driving $\left(\delta_{\phi} \rightarrow 0\right)$ the level transitions in the dynamics are dictated by the dynamic phase. However, in the case of nonadiabatic driving the geometric phase can affect the transitions and lead to nontrivial phenomena.

As in the previous section we study the evolution after the rotation is switched on suddenly at $t=0$, but now we assume the system to be prepared in the exact ground state of the time-independent Dicke Hamiltonian $\hat{H}_{\mathrm{D}}$. Moreover, we now calculate the time evolution of the mean photon number using Eq. (52). The coefficients $\chi_{k}$ are calculated numerically for the initial condition $\chi_{l}(0)=\delta_{l, 1}$ and used for evaluating quantities like the photon number,

$$
n_{\mathrm{ph}}(t)=\frac{1}{j} \sum_{k, l} \chi_{k}^{*}(t) \chi_{l}(t) e^{i \mathcal{E}_{k l}(t)-i \gamma_{k l}(t)}\left\langle\varphi_{k}(0)\left|\hat{a}^{\dagger} \hat{a}\right| \varphi_{l}(0)\right\rangle .
$$

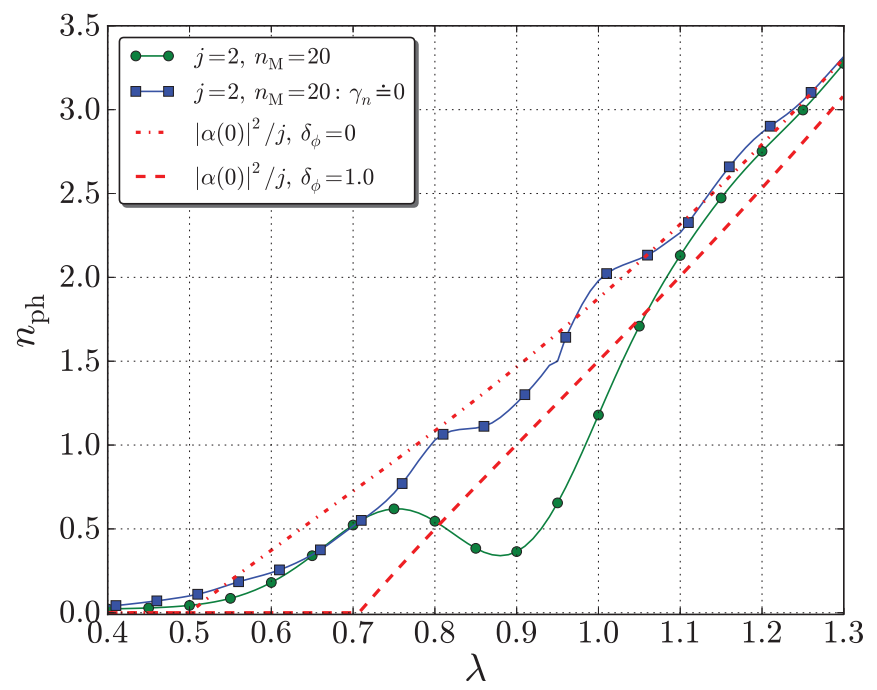

FIG. 10. (Color online) Mean photon number after a single period of revolution $T_{\phi}$ as a function of the coupling and for $\omega=\omega_{0}=\delta_{\phi}=$ 1. Solid lines were obtained by numerically solving Eq. (52) and using Eq. (53) for $j=2$ with $n_{\mathrm{M}}=20$. The (blue) line with squares was calculated by setting all the geometric phases to $0, \gamma_{l}=0, \forall l=$ $1,2, \ldots,(2 j+1)\left(n_{\mathrm{M}}+1\right)$. Dashed lines represent mean-field values for $\hat{H}_{\mathrm{D}}$ and $\hat{H}_{\mathrm{ROT}}$, respectively.

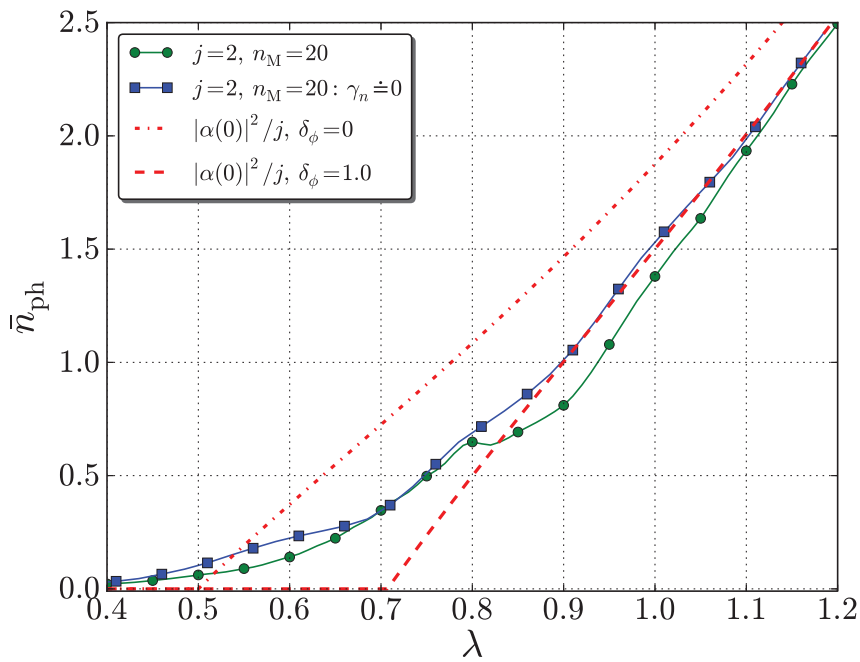

FIG. 11. (Color online) Coupling dependence of the mean photon number averaged over a time $T=10 T_{\phi}$ and for $\omega=\omega_{0}=\delta_{\phi}=1$. Solid lines were obtained by numerically solving Eq. (52) and timeaveraging Eq. (53) for $j=2$ and $n_{\mathrm{M}}=20$. The (blue) line with squares was calculated by setting all the geometric phases to 0 .

We also get

$$
A_{l k}(\phi)=-\delta_{\phi}\left\langle\phi_{l}(0)\left|\hat{J}_{z}\right| \phi_{k}(0)\right\rangle
$$

and therefore a geometric phase,

$$
\gamma_{l}(t)=\int_{0}^{t} d \tau A_{l l}(\tau)=-\left\langle\varphi_{l}(0)\left|\hat{J}_{z}\right| \varphi_{l}(0)\right\rangle \phi(t) .
$$

We have studied the influence of the geometric phases $\gamma_{l}$ on the dynamics by calculating the mean photon number using Eq. (52) and Eq. (53), once with the values of $\gamma_{l}$ given in Eq. (55) and once for $\gamma_{l}=0$ for all levels, $l=1, \ldots,(2 j+$ $1)\left(n_{\mathrm{M}}+1\right)$. The results for $n_{\mathrm{ph}}\left(T_{\phi}\right)$, shown in Fig. 10, clearly indicate that the minimum of the full solution [(green) line with circles] disappears if the geometric phases are artificially set to 0 [(blue) line with squares]. The same effect is seen in the timeaveraged photon number, illustrated in Fig. 11, where the darkening is less pronounced. Here we noticed also a difference between the results obtained with and those without geometric phases, at small values of $\lambda$. We attribute this disparity to finitesize effects, which are expected to be most pronounced for weak coupling. Unfortunately, calculations based on Eq. (52) are hardly practicable for $j>2$. Therefore we were not able to study the size dependence using this method.

The results in Figs. 10 and 11 show that the geometric phases play an important role in the region slightly above the critical point $\lambda_{c}$. In fact, for both $\lambda_{c}^{0}<\lambda<\lambda_{c}$ and $\lambda \gg \lambda_{c}$ their effect on the photon density seems to be, at most, marginal. The fact that the minimum disappears if the geometric phases are quenched suggests that these phases are instrumental in the dynamically generated darkness.

\section{CONCLUSION}

We have studied the dynamics of a rotationally driven version of the Dicke model, where the collective spin is rotated with a constant velocity about a fixed axis. The main aim was to calculate the effect of the imposed drive on important 
quantities such as the mean photon number and the atomic inversion. We have used both a numerical technique for the exact quantum dynamics of a finite number of two-level atoms and the time-dependent mean-field theory, which is expected to be valid in the thermodynamic limit. The results obtained with these two methods are consistent with each other, although the relatively small size treated numerically does not allow us to draw a definitive conclusion about the limiting behavior of the exact quantum dynamics for arbitrary large system sizes.

Most calculations have been carried out within a corotating frame, where the dynamics is governed by a time-independent Dicke model with a renormalized level splitting. In this frame there exists a ground state which undergoes a transition as a function of the coupling strength from a normal phase with vanishing photon number to a superradiant phase with an increasing number of photons (as well as a growing atomic inversion). The critical coupling strength is higher than that for the undriven Dicke model and increases with the driving velocity. In the unrotated frame the expectation value of the collective spin precesses around the static field above the critical point, with the frequency of the drive.

We have studied in great detail the quench dynamics of the driven Dicke model, where the system is prepared in the mean-field ground state of the undriven Hamiltonian and then suddenly experiences the rotation. We found a remarkable dynamically generated darkness, a reduction of the photon number due to the rotational driving, both in the numerical calculations for the quantum evolution and in the classical dynamics of the mean-field theory. We have interpreted this phenomenon in two ways. On the one hand, we have attributed it to a nonlinear slowing-down of the classical dynamical system representing time-dependent mean-field theory. On the other hand, for a small number of two-level atoms, where the evolution has to be treated quantum mechanically, we identified the geometric phase of the instantaneous eigenstates as the main actor producing the darkening. It would be very interesting to explore possible connections between the two interpretations. Thus one may wonder about the fate of the geometric phase in the mean-field limit. For a very simple quantum-mechanical system the geometric phase has been related to the shift of angle variables in the classical limit [30], known as the Hannay angle [31]. This angle has been introduced in the context of slowly (adiabatically) cycled integrable systems and therefore cannot be transferred immediately to our model. Future studies may find out whether some classical analog for the geometric phases of quantum dynamics (Berry phases) is responsible for the dynamically generated darkness reported here.

\section{ACKNOWLEDGMENTS}

V.G. is grateful to KITP for hospitality. This work was supported by the Swiss National Science Foundation.

\section{APPENDIX A: SOLUTION OF THE INITIAL VALUE PROBLEM FOR THE LINEARIZED DYNAMICAL SYSTEM}

We consider the classical dynamical system in Sec. IV C and assume the initial deviations from equilibrium- $Q(0)$ and $q(0)$ for the normal phase and $X(0)$ and $x(0)$ for the superradiant phase - to be small, together with $P(0)=p(0)=$ 0 . This allows us to use the linearized equations of motion.

\section{Normal phase: $\lambda<\lambda_{c}$}

For $\lambda<\lambda_{c}$ the linearized equations of motion are given by Eq. (37). In view of the initial conditions $P(0)=p(0)=0$ the general solution is

$$
\begin{aligned}
& Q(t)=A_{+} \cos \left(\varepsilon_{+} t\right)+A_{-} \cos \left(\varepsilon_{-} t\right), \\
& q(t)=a_{+} \cos \left(\varepsilon_{+} t\right)+a_{-} \cos \left(\varepsilon_{-} t\right),
\end{aligned}
$$

with eigenmode frequencies $\varepsilon_{ \pm}$given by Eq. (38). Equations (37) imply the following relation between coefficients:

$$
a_{ \pm}=f_{ \pm} A_{ \pm}, \quad \text { with } \quad f_{ \pm}=\frac{\varepsilon_{ \pm}^{2}-\Omega^{2}}{2 \lambda \Omega} .
$$

The initial conditions yield the amplitudes

$$
A_{+}=\frac{q(0)-Q(0) f_{-}}{f_{+}-f_{-}}, \quad A_{-}=\frac{Q(0) f_{+}-q(0)}{f_{+}-f_{-}} .
$$

\section{Superradiant phase: $\lambda>\lambda_{c}$}

For $\lambda>\lambda_{c}$ the linearized equations of motion, (41), describe the deviations $X(t)=Q(t)-Q_{0}$ and $x(t)=q(t)-q_{0}$ from the stationary state $\left(Q_{0}, q_{0}\right)$. The general solution for $P(0)=p(0)=0$ is

$$
\begin{gathered}
X(t)=A_{+} \cos \left(\varepsilon_{+} t\right)+A_{-} \cos \left(\varepsilon_{-} t\right), \\
x(t)=a_{+} \cos \left(\varepsilon_{+} t\right)+a_{-} \cos \left(\varepsilon_{-} t\right),
\end{gathered}
$$

with eigenmode frequencies given by Eq. (42). Using Eqs. (41) we find the following relation between coefficients,

$$
a_{ \pm}=g_{ \pm} A_{ \pm}, \quad g_{ \pm}=\frac{2 \sqrt{2} \omega \lambda_{c}^{2}}{\left(\varepsilon_{ \pm}^{2}-\omega^{2}\right) \sqrt{\lambda^{2}+\lambda_{c}^{2}}},
$$

while the initial conditions imply

$$
A_{+}=\frac{x(0)-X(0) g_{-}}{g_{+}-g_{-}}, \quad A_{-}=\frac{X(0) g_{+}-x(0)}{g_{+}-g_{-}} .
$$

For $\lambda \gg \lambda_{c}$ the coefficient $g_{+}$is very small and $x(t) \approx$ $a_{-} \cos \left(\varepsilon_{-} t\right)$, where $\varepsilon_{-} \approx \omega$.

\section{Mean photon number}

It is now straightforward to calculate some important quantities such as the mean photon number. For simplicity we only consider values averaged over long times and calculate

$$
\bar{n}_{\mathrm{ph}}=\lim _{T \rightarrow \infty} \frac{1}{2 T} \int_{0}^{T} d t\left[q^{2}(t)+p^{2}(t)\right] .
$$

We choose the initial conditions in Sec. V; i.e., $Q(0)$ and $q(0)$ correspond to the stationary state of the time-independent Dicke model. We limit ourselves to the nontrivial case $\lambda>\lambda_{c}^{0}$, where

$$
\begin{aligned}
& Q(0)= \pm \sqrt{2}\left(1-\frac{\left[\lambda_{c}^{0}\right]^{2}}{\lambda^{2}}\right)^{\frac{1}{2}}, \\
& q(0)=\mp \frac{2 \lambda}{\omega}\left(1-\frac{\left[\lambda_{c}^{0}\right]^{4}}{\lambda^{4}}\right)^{\frac{1}{2}} .
\end{aligned}
$$




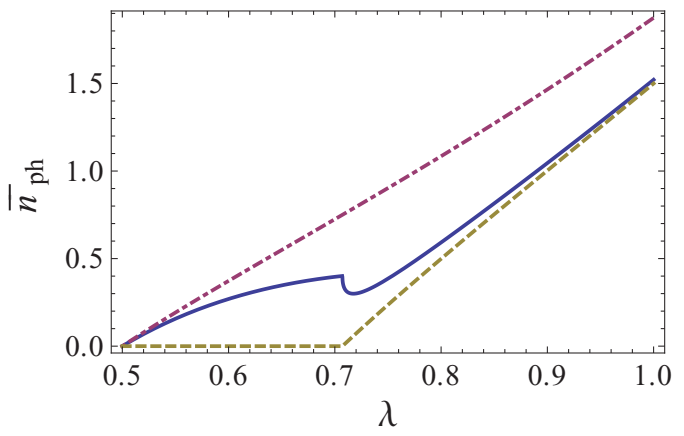

FIG. 12. (Color online) Mean photon number in the linear limit for initial conditions corresponding to the ground state of the time-independent Dicke Hamiltonian and for a subsequent evolution governed by the time-dependent Dicke Hamiltonian with parameters $\omega=\omega_{0}=1, \delta_{\phi}=1$.

For $\lambda_{c}^{0}<\lambda<\lambda_{c}$ the system evolves in the normal phase of the time-dependent Dicke model, where the stationary values of the coordinates vanish, $Q_{0}=q_{0}=0$. We obtain

$$
\bar{n}_{\mathrm{ph}}=\frac{1}{4 \omega^{2}}\left[\left(\varepsilon_{+}^{2}+\omega^{2}\right) a_{+}^{2}+\left(\varepsilon_{-}^{2}+\omega^{2}\right) a_{-}^{2}\right],
$$

where the eigenvalues $\varepsilon_{ \pm}$and coefficients $f_{ \pm}$are given by Eqs. (38) and (A3), respectively.

For $\lambda>\lambda_{c}$, i.e., in the superradiant phase of the timedependent Dicke model, the photon number of the ground state is nonzero and the stationary coordinates $Q_{0}$ and $q_{0}$ are given by Eq. (39). The time-dependent coordinates are $q(t)=q_{0}+x(t)$ and $Q(t)=Q_{0}+X(t)$. Therefore the photon number averaged over a long time $T$ is

$$
\bar{n}_{\mathrm{ph}}=\frac{1}{2} q_{0}^{2}+\frac{1}{4 \omega^{2}}\left[\left(\varepsilon_{+}^{2}+\omega^{2}\right) a_{+}^{2}+\left(\varepsilon_{-}^{2}+\omega^{2}\right) a_{-}^{2}\right],
$$

where $\varepsilon_{ \pm}$and $a_{ \pm}$are defined by Eqs. (42) and (A6), respectively, and $x(0)=q(0)-q_{0}, X(0)=Q(0)-Q_{0}$. The result shown in Fig. 12 is in qualitative agreement with that obtained with the full Hamiltonian (Fig. 5), although the linear approximation cannot be trusted for $\lambda$ of the order of $\lambda_{c}$, where the potential is strongly nonlinear.

\section{APPENDIX B: SLOWING-DOWN IN THE MEXICAN HAT}

To illustrate the dynamically generated darkness detected for a finite rotation velocity above the critical point of the Dicke model (Sec. V), we consider a classical system of 2 degrees of freedom described by the Lagrangian

$$
L=\frac{m}{2}\left(\dot{q}_{1}^{2}+\dot{q}_{2}^{2}\right)+\frac{k}{2}\left(q_{1}^{2}+q_{2}^{2}\right)-\frac{g}{4}\left(q_{1}^{2}+q_{2}^{2}\right)^{2},
$$

where $k>0, g>0$. In polar coordinates, $q_{1}=\rho \cos \varphi, q_{2}=$ $\rho \sin \varphi$, Eq. (B1) reads

$$
L=\frac{m}{2}\left(\dot{\rho}^{2}+\rho^{2} \dot{\varphi}^{2}\right)-V(\rho),
$$

where

$$
V(\rho)=-\frac{k}{2} \rho^{2}+\frac{g}{4} \rho^{4}
$$

has a minimum $V_{\min }=-k^{2} /(4 g)$ at $\rho_{0}=\sqrt{k / g}$. For initial conditions $\dot{q}_{1}(0)=\dot{q}_{2}(0)=0$ a particle moving in this

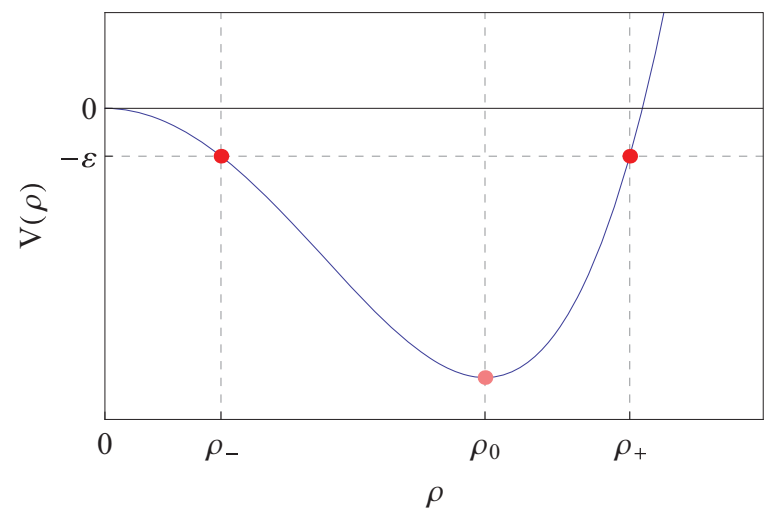

FIG. 13. (Color online) Potential as a function of the radius $\rho$. For a negative energy $-\varepsilon$, the motion is limited by turning points $\rho_{ \pm}$ and does not reach the origin.

"Mexican hat" executes radial motions, around $\rho_{0}$ if the energy is negative and centered at $\rho=0$ if the energy is positive. In either case the particle spends a lot of time at small values of $\rho$ if the energy is low. To see this quantitatively we consider the special case of a negative energy (for $\dot{\varphi}=0$ ),

$$
\frac{m}{2} \dot{\rho}^{2}-\frac{k}{2} \rho^{2}+\frac{g}{4} \rho^{4}=-\varepsilon,
$$

with $\varepsilon>0$, where the particle moves between turning points

$$
\rho_{ \pm}^{2}=\rho_{0}^{2} \pm \sqrt{\rho_{0}^{4}-\frac{4 \varepsilon}{g}}
$$

as illustrated in Fig. 13.

Equation (B4) then implies

$$
d t= \pm \sqrt{\frac{2 m}{g}} \frac{d \rho}{\sqrt{\left(\rho^{2}-\rho_{-}^{2}\right)\left(\rho_{+}^{2}-\rho^{2}\right)}} .
$$

The time $T$ for moving from $\rho_{-}$to $\rho_{+}$(or the other way) is

$$
\begin{aligned}
T & =\sqrt{\frac{2 m}{g}} \int_{\rho_{-}}^{\rho_{+}} \frac{d \rho}{\sqrt{\left(\rho^{2}-\rho_{-}^{2}\right)\left(\rho_{+}^{2}-\rho^{2}\right)}} \\
& =\sqrt{\frac{2 m}{g}} \frac{1}{\rho_{+}} K\left(\frac{\sqrt{\rho_{+}^{2}-\rho_{-}^{2}}}{\rho_{+}}\right),
\end{aligned}
$$

and similarly, we obtain

$$
\begin{aligned}
\int_{0}^{T} d t \rho^{2}(t) & =\sqrt{\frac{2 m}{g}} \int_{\rho_{-}}^{\rho_{+}} \frac{\rho^{2} d \rho}{\sqrt{\left(\rho^{2}-\rho_{-}^{2}\right)\left(\rho_{+}^{2}-\rho^{2}\right)}} \\
& =\sqrt{\frac{2 m}{g}} \rho_{+} E\left(\frac{\sqrt{\rho_{+}^{2}-\rho_{-}^{2}}}{\rho_{+}}\right),
\end{aligned}
$$

where $E(k)$ and $K(k)$ are complete elliptic integrals. Therefore the time average of $\rho^{2}$ is given by

$$
\left\langle\rho^{2}\right\rangle_{T}:=\frac{1}{T} \int_{0}^{T} d t \rho^{2}(t)=\frac{E\left(\frac{\sqrt{\rho_{+}^{2}-\rho_{-}^{2}}}{\rho_{+}}\right)}{K\left(\frac{\sqrt{\rho_{+}^{2}-\rho_{-}^{2}}}{\rho_{+}}\right)} \rho_{+}^{2} .
$$


For $\varepsilon \rightarrow 0$ the turning point $\rho_{-}$moves to 0 and the elliptic integrals have the limiting behavior

$$
\begin{aligned}
& E\left(\frac{\sqrt{\rho_{+}^{2}-\rho_{-}^{2}}}{\rho_{+}}\right) \sim 1, \\
& K\left(\frac{\sqrt{\rho_{+}^{2}-\rho_{-}^{2}}}{\rho_{+}}\right) \sim \log \left(\frac{4 \rho_{+}}{\rho_{-}}\right) .
\end{aligned}
$$

It follows that the average value of $\rho^{2}(t)$ tends to 0 if $\varepsilon$ tends to 0 , i.e., if the initial potential energy coincides with the hump at $\rho=0$. A similar result is obtained for the average $\left\langle p_{\rho}^{2}\right\rangle_{T}=m^{2}\left\langle\dot{\rho}^{2}\right\rangle_{T}$, which also tends to 0 (logarithmically) for $\varepsilon \rightarrow 0$. The origin of this behavior is simply that with decreasing energy the particle slows down more and more upon approaching the turning point $\rho_{-}$. At the critical energy (here $\varepsilon=0$ ), where the motion changes from a simple oscillation about a minimum (for $\varepsilon<0$ ) to a motion over the potential hump (for $\varepsilon>0$ ), the time span for a single period diverges and both $\left\langle\rho^{2}\right\rangle_{T}$ and $\left\langle p_{\rho}^{2}\right\rangle_{T}$ tend to 0 . Figure 14 shows both the analytical expression, (B9), and the numerical results for

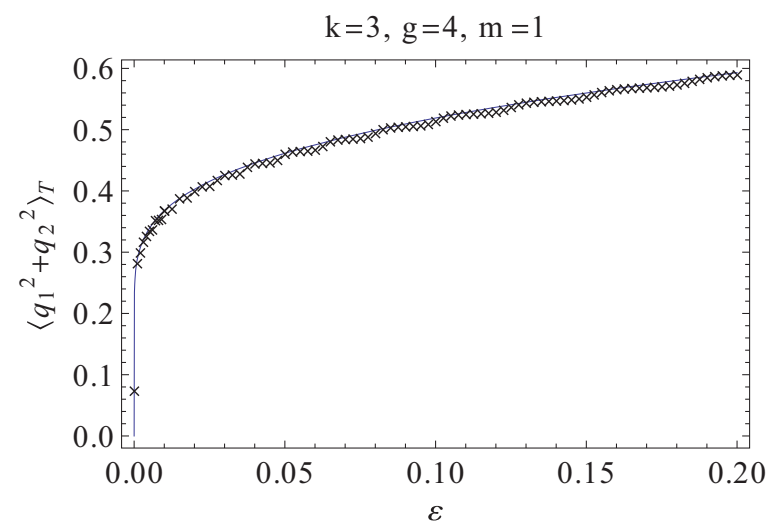

FIG. 14. (Color online) Time average of $q_{1}^{2}(t)+q_{2}^{2}(t)$ at negative energies $-\varepsilon$ for the isotropic "Mexican hat" potential, (B3), with parameters $m=1, k=3, g=4$. The solid line is the analytical solution; crosses represent the numerical integration of the equations of motion.

$\left\langle\rho^{2}\right\rangle_{T}$. The agreement is excellent and serves as a test for the algorithm used in the numerical calculations.
[1] R. H. Dicke, Phys. Rev. 93, 99 (1954).

[2] M. Tavis and F. W. Cummings, Phys. Rev. 170, 379 (1968); M. T. Tavis, Ph.D. thesis, University of California, Riverside, 1968, arXiv:1206.0078.

[3] K. Hepp and E. H. Lieb, Ann. Phys. 76, 360 (1973).

[4] Y. K. Wang and F. T. Hioe, Phys. Rev. A 7, 831 (1973).

[5] H. J. Carmichael, C. W. Gardiner, and D. F. Walls, Phys. Lett. A 46, 47 (1973).

[6] N. Lambert, C. Emary, and T. Brandes, Phys. Rev. Lett. 92, 073602 (2004); S.-B. Zheng, Phys. Rev. A 84, 033817 (2011).

[7] C. Emary and T. Brandes, Phys. Rev. E 67, 066203 (2003).

[8] K. Rzazewski, K. Wódkiewicz, and W. Zakowicz, Phys. Rev. Lett. 35, 432 (1975).

[9] J. Keeling, J. Phys.: Condens. Matter 19, 295213 (2007).

[10] A. Vukics and P. Domokos, Phys. Rev. A 86, 053807 (2012).

[11] F. Dimer, B. Estienne, A. S. Parkins, and H. J. Carmichael, Phys. Rev. A 75, 013804 (2007).

[12] F. Brennecke, T. Donner, S. Ritter, T. Bourdel, M. Köhl, and T. Esslinger, Nature 450, 268 (2007).

[13] K. Baumann, C. Guerlin, F. Brennecke, and T. Esslinger, Nature (London) 464, 1301 (2010).

[14] A. Altland and F. Haake, Phys. Rev. Lett. 108, 073601 (2012).

[15] A. Altland, V. Gurarie, T. Kriecherbauer, and A. Polkovnikov, Phys. Rev. A 79, 042703 (2009).

[16] A. P. Itin and P. Törmä, Phys. Rev. A 79, 055602 (2009).
[17] V. M. Bastidas, C. Emary, B. Regler, and T. Brandes, Phys. Rev. Lett. 108, 043003 (2012).

[18] E. A. Yuzbashyan, V. B. Kuznetsov, and B. L. Altshuler, Phys. Rev. B 72, 144524 (2005); E. A. Yuzbashyan, O. Tsyplyatyev, and B. L. Altshuler, Phys. Rev. Lett. 96, 097005 (2006).

[19] O. Tsyplyatyev and D. Loss, Phys. Rev. B 82, 024305 (2010); Phys. Rev. A 80, 023803 (2009).

[20] Ch. Sträter, O. Tsyplyatyev, and A. Faribault, Phys. Rev. B 86, 195101 (2012).

[21] F. Plastina, G. Liberti, and A. Carollo, Europhys. Lett. 76, 182 (2006).

[22] H. Tal-Ezer and R. Kosloff, J. Chem. Phys. 81, 3967 (1984).

[23] O. Castaños, E. Nahmad-Achar, R. López-Peña, and J. G. Hirsch, Phys. Rev. A 83, 051601 (2011).

[24] W. Zhang, D. H. Feng, and R. Gilmore, Rev. Mod. Phys. 62, 867 (1990).

[25] D. V. Kapor, M. J. Škrinjar, and S. D. Stojanović, Phys. Rev. B 44, 2227 (1991).

[26] M. A. M. de Aguiar, K. Furuya, C. H. Lewenkopf, and M. C. Nemes, Ann. Phys. (NY) 216, 291 (1992).

[27] M. Tomka, A. Polkovnikov, and V. Gritsev, Phys. Rev. Lett. 108, 080404 (2012).

[28] D. Xiao, M.-C. Chang, and Q. Niu, Rev. Mod. Phys. 82, 1959 (2010)

[29] M. V. Berry, Proc. R. Soc. A 392, 45 (1984).

[30] M. V. Berry, J. Phys. A 18, 15 (1985).

[31] J. H. Hannay, J. Phys. A 18, 221 (1985). 